This item was submitted to Loughborough's Research Repository by the author.

Items in Figshare are protected by copyright, with all rights reserved, unless otherwise indicated.

\title{
Elastic waves trapped above a cylindrical cavity
}

PLEASE CITE THE PUBLISHED VERSION

https://doi.org/10.1137/17M1155296

\section{PUBLISHER}

(c) Society for Industrial and Applied Mathematics

\section{VERSION}

AM (Accepted Manuscript)

\section{PUBLISHER STATEMENT}

This work is made available according to the conditions of the Creative Commons Attribution-NonCommercialNoDerivatives 4.0 International (CC BY-NC-ND 4.0) licence. Full details of this licence are available at: https://creativecommons.org/licenses/by-nc-nd/4.0/

\section{LICENCE}

CC BY-NC-ND 4.0

\section{REPOSITORY RECORD}

Linton, C.M., and lan Thompson. 2019. "Elastic Waves Trapped Above a Cylindrical Cavity". figshare. https://hdl.handle.net/2134/33624. 


\title{
ELASTIC WAVES TRAPPED ABOVE A CYLINDRICAL CAVITY
}

\author{
C. M. LINTON* AND I. THOMPSON ${ }^{\dagger}$
}

\begin{abstract}
The existence of trapped elastic waves above a circular cylindrical cavity in a halfspace is demonstrated. These modes propagate parallel to the cylinder and their amplitude decays exponentially as the observer moves away from it. Dispersion relations connecting the frequency with the wavenumber along the cylinder are obtained using an analytical technique based on multipole expansions, and solved numerically. Critical frequencies at which modes cut on and off are determined and a range of contour plots illustrating the displacement fields are presented.
\end{abstract}

Key words. Elastic waves, Trapped modes, Wave diffraction, Multipole expansions

AMS subject classifications. 74B05,74J05,74J15,74J20

1. Introduction. We consider an elastic half-space containing a horizontal circular cylindrical cavity and determine whether trapped modes can propagate along the cavity. We take the $y$-axis to be aligned with the cylinder, so that the modes have the form $\mathbf{U}(x, z, t) \mathrm{e}^{\mathrm{i} \ell y}$ for some real $\ell$ and a vector-valued function $\mathbf{U}$. The modes are localised in the sense that $\mathbf{U}(x, z, t) \rightarrow \mathbf{0}$ as $x^{2}+z^{2} \rightarrow \infty$ and the energy (per unit length of cylinder) is finite. The method we employ is an extension of the multipole expansion technique developed in [13] for the scattering problem in which a Rayleigh wave is obliquely incident on a cylindrical cavity. This geometry is relevant to the case of a tunnel embedded in a half-space and simlar techniques have been used for related problems in, e.g., [12, 21].

An unbounded homogeneous elastic medium supports longitudinal (pressure, primary or P-) waves with wavenumber $k_{1}$ and transverse (shear, secondary or S-) waves with wavenumber $k_{2}$. A homogeneous half-space supports Rayleigh waves on its free surface with wavenumber $k_{\mathrm{R}}$. The wavenumbers depend on the properties of the elastic material but always satisfy $0<k_{1}<k_{2}<k_{\mathrm{R}}$. Our strategy is to look for trapped modes with $\ell>k_{\mathrm{R}}$, because in this regime there are no longitudinal, transverse or Rayleigh waves, meaning that propagation to infinity away from the cylinder is not possible. Our main objective is to find a dispersion relation between possible values of $\ell$ and the frequency $\omega$, which is considered fixed. Mathematically, trapped modes correspond to discrete eigenvalues of the associated differential operator, for which the continuous spectrum is $0<\ell \leq k_{\mathrm{R}}$. Our analysis does not attempt to determine whether eigenvalues embedded in this continuous spectrum can exist.

A natural analogue to the problem under consideration here appears in the linear theory of water waves. In that context, the existence of a trapped mode above a submerged, horizontal, rigid circular cylinder in infinitely deep water was first established by Ursell [18] for small cylinders and then more generally by Jones [11] and others $[19,1]$. Computations of the dispersion curves for trapped modes were first performed in [14], showing that more and more modes appear as the depth of submergence decreases, though the number of modes is always finite.

Unlike the case of a rigid cylinder in a fluid that extends to infinity in all directions, a cylindrical cavity in an otherwise unbounded elastic medium supports modes which travel along its surface, parallel to the cylinder axis. For circular cylinders, dispersion curves were computed in $[4,5]$, but modes of this type have been shown to exist for

\footnotetext{
* Department of Mathematical Sciences, Loughborough University, Leicestershire, LE11 3TU, UK

${ }^{\dagger}$ Department of Mathematical Sciences, University of Liverpool, Liverpool L69 7ZL, UK
} 
cylinders of arbitrary cross section [6,3]. These exist for values of $\ell$ in the range $k_{2}<\ell<k_{\mathrm{R}}$ and are therefore separated from the phenomena studied in this article, but we easily recover their dispersion relations from our analysis.

The plan of the paper is as follows. In $\S 2$ we formulate the boundary-value problem corresponding to a cavity in a half-space with $\ell>k_{\mathrm{R}}$, using the Helmholtz decomposition for the displacement field. This leads to a representation of the solution in terms of three scalar fields, each of which satisfies a Helmholtz equation. These scalar fields are expanded as series of multipoles (each of which is a singular wavefunction, constructed to satisfy the stress-free condition on the flat free surface) and the application of the boundary conditions on the cavity wall then leads to an infinite system of linear, algebraic equations which can be solved numerically by truncation. In $\S 3$ we show how the symmetry of the geometry can be used to divide possible modes into two distinct categories and in $\S 4$ we show how to recover the dispersion relations found in $[4,5]$ for the case of a cavity in an otherwise unbounded medium. The limit $\ell \rightarrow k_{\mathrm{R}}$ is considered in $\S 5$. This allows us to accurately compute the points in parameter space at which modes cut on and off and is also used in $\S 6$ to enable us to make some observations about the nature of the modes themselves and the parameter regimes in which they are found. Our numerical results are presented in $\S 7$ and some concluding remarks are made in $\S 8$. Technical details concerning the construction of the multipoles and their behaviour in the limit $\ell \rightarrow k_{\mathrm{R}}$ are deferred to the appendix.

2. Formulation. The region $z<0$ is a homogeneous and isotropic elastic medium containing a cylindrical cavity of radius $a$, aligned with the $y$-axis. Unit vectors in the $x$-, $y$ - and $z$-directions are denoted by $\mathbf{e}_{x}, \mathbf{e}_{y}$ and $\mathbf{e}_{z}$, respectively. We introduce polar coordinates centred at the point $(x, z)=(0,-h)$ via

$$
r \sin \theta=x \quad \text { and } \quad r \cos \theta=-(z+h),
$$

so that $\theta$ is measured anticlockwise from the downward vertical. The cavity is the region $r<a$ and the elastic medium occupies the space $\Omega \times \mathbb{R}$, where $\Omega=\{(x, z)$ : $z \leq 0$ and $r \geq a\}$.

We consider time-harmonic motion with frequency $\omega$ and assume and suppress an $\mathrm{e}^{-\mathrm{i} \omega t}$ dependence throughout. The displacement field then satisfies Navier's equation

$$
c_{1}^{2} \nabla(\nabla \cdot \mathbf{u})-c_{2}^{2} \nabla \times(\nabla \times \mathbf{u})=-\omega^{2} \mathbf{u} .
$$

Here

$$
c_{1}^{2}=(\lambda+2 \mu) / \rho, \quad c_{2}^{2}=\mu / \rho,
$$

in which $\lambda$ and $\mu$ are the Lamé constants related to the Young's modulus $E$ and Poisson's ratio $\sigma$ via $\lambda=E \sigma /[(1-2 \sigma)(1+\sigma)], \mu=E /[2(1+\sigma)]$ and $\rho$ is the density of the elastic medium. For most materials $0<\sigma<1 / 2$, but $-1<\sigma \leq 0$ is also possible. Note that $\lambda+\mu=E /[2(1+\sigma)(1-2 \sigma)]>0$ and so we always have $c_{1}^{2}>c_{2}^{2}$. The quantity $c_{1}$ is the wave speed for longitudinal (pressure, primary or P-) waves in an infinite medium, whereas $c_{2}$ is the speed of transverse (shear, secondary or S-) waves. We introduce corresponding wavenumbers $k_{i}=\omega / c_{i}$ and, since the speeds $c_{i}$ are assumed fixed, we will often simply use the word frequency to refer to the numbers $k_{i}$. The ratio

$$
\Lambda=\frac{k_{1}^{2}}{k_{2}^{2}}=\frac{c_{2}^{2}}{c_{1}^{2}}=\frac{1-2 \sigma}{2(1-\sigma)}
$$


does not depend on $E$ and we have $0<\Lambda<3 / 4$. Note that $\rho \omega^{2}=\mu k_{2}^{2}$ and $\lambda+2 \mu=$ $\mu / \Lambda$.

We wish to investigate solutions to (2.2), subject to stress-free boundary conditions on both $z=0$ and $r=a$. In order to represent physically viable trapped modes, we set $\mathbf{u}=\mathrm{e}^{\mathrm{i} \ell y} \tilde{\mathbf{u}}(x, z)$ and require that $\tilde{\mathbf{u}}(x, z) \rightarrow \mathbf{0}$ as $r \rightarrow \infty$, and that the integral

$$
\int_{\Omega}|\tilde{\mathbf{u}}|^{2} \mathrm{~d} S
$$

which is proportional to the energy per unit length of the wave, is finite. This problem can be written in an abstract form (as in [2] for a related problem) as $A(\ell) \tilde{\mathbf{u}}=\omega^{2} \tilde{\mathbf{u}}$, where $A$ is an unbounded self-adjoint operator which encapsulates the differential equation (2.2) and associated boundary conditions. In such a formulation the wavenumber $\ell$ is a parameter, and possible values of $\omega$ correspond to eigenvalues of $A$. The existence of eigenvalues is not guaranteed, because the resolvent of $A$ is not compact. Our approach is to fix $\omega$ and construct solutions using multipole expansions, each of which is a solution to Navier's equation which is singular at $r=0$ and which satisfies the stress-free boundary conditions on $z=0$. Application of the boundary conditions on $r=a$ then leads to an infinite system of linear equations in the form $K(\ell) \mathbf{x}=\mathbf{0}$. We truncate the infinite matrix $K$ and seek those values of $\ell$ for which the determinant of the resulting finite matrix vanishes. The associated eigenvector $\mathbf{x}$ is then made up of the coefficients in the original multipole expansion.

We utilise the Helmholtz decomposition of the displacement field

$$
\mathbf{u}=\nabla \Phi+\nabla \times \mathbf{\Psi}, \quad \nabla \cdot \mathbf{\Psi}=0
$$

with

$$
\Phi=\phi(x, z) \mathrm{e}^{\mathrm{i} \ell y}, \quad \Psi=\psi(x, z) \mathrm{e}^{\mathrm{i} \ell y} \mathbf{e}_{y}+\frac{\mathrm{i} \ell}{k_{2}^{2}} \nabla\left(\psi(x, z) \mathrm{e}^{\mathrm{i} \ell y}\right)+\frac{1}{k_{2}} \nabla \times\left(\chi(x, z) \mathrm{e}^{\mathrm{i} \ell y} \mathbf{e}_{y}\right)
$$

and then $\phi, \psi$ and $\chi$ satisfy the two-dimensional scalar Helmholtz equations

$$
\left(\nabla_{x z}^{2}-q_{1}^{2}\right) \phi=0, \quad\left(\nabla_{x z}^{2}-q_{2}^{2}\right) \psi=\left(\nabla_{x z}^{2}-q_{2}^{2}\right) \chi=0 .
$$

For convenience we have defined

$$
q_{i}^{2}=\ell^{2}-k_{i}^{2}
$$

and $\nabla_{x z}^{2} \equiv \partial_{x}^{2}+\partial_{z}^{2}$. We will always have $q_{i}^{2}>0$ and choose to take $q_{i}>0$. Note that the second term in the expression for $\boldsymbol{\Psi}$ in (2.6) plays no role in what follows since it does not contribute to $\mathbf{u}$; it is there purely to make $\boldsymbol{\Psi}$ divergence free. The particular form of (2.6), which leads to convenient expressions for the velocity components in cylindrical coordinates, follows that used in [5]. In this formulation, $\phi$ represents the irrotational component of the field while, $\psi$ and $\chi$ together specify the divergence-free component, according to (2.6). We also have

$$
\tilde{\mathbf{u}}=\left(\phi_{x}-\psi_{z}+\frac{\mathrm{i} \ell}{k_{2}} \chi_{x}\right) \mathbf{e}_{x}+\left(\mathrm{i} \ell \phi-\frac{q_{2}^{2}}{k_{2}} \chi\right) \mathbf{e}_{y}+\left(\phi_{z}+\psi_{x}+\frac{\mathrm{i} \ell}{k_{2}} \chi_{z}\right) \mathbf{e}_{z}
$$

and

$$
\nabla \cdot \mathbf{u}=-k_{1}^{2} \phi \mathrm{e}^{\mathrm{i} \ell y}
$$


On $z=0$ we require zero traction, which in turn means that the components $\tau_{13}$, $\tau_{23}$ and $\tau_{33}$ of the stress tensor must vanish there. Now

$$
\begin{aligned}
& \tau_{13}=\mu \mathrm{e}^{\mathrm{i} \ell y}\left(2 \phi_{x z}+\psi_{x x}-\psi_{z z}+\frac{2 \mathrm{i} \ell}{k_{2}} \chi_{x z}\right), \\
& \tau_{23}=\mu \mathrm{e}^{\mathrm{i} \ell y}\left(2 \mathrm{i} \ell \phi_{z}+\mathrm{i} \ell \psi_{x}+\frac{\nu^{2}}{k_{2}} \chi_{z}\right), \\
& \tau_{33}=\mathrm{e}^{\mathrm{i} \ell y}\left(-\lambda k_{1}^{2} \phi+2 \mu\left(\phi_{z z}+\psi_{x z}+\frac{\mathrm{i} \ell}{k_{2}} \chi_{z z}\right)\right),
\end{aligned}
$$

where we have written

$$
\nu^{2}=-q_{2}^{2}-\ell^{2}=k_{2}^{2}-2 \ell^{2},
$$

and so

$$
\begin{aligned}
2\left(\phi_{x z}+\psi_{x x}\right)-q_{2}^{2} \psi+\frac{2 \mathrm{i} \ell}{k_{2}} \chi_{x z}=0 & \text { on } z=0, \\
2 \mathrm{i} \ell \phi_{z}+\mathrm{i} \ell \psi_{x}+\frac{\nu^{2}}{k_{2}} \chi_{z}=0 & \text { on } z=0, \\
2\left(\psi_{x z}-\phi_{x x}\right)-\nu^{2} \phi+\frac{2 \mathrm{i} \ell}{k_{2}} \chi_{z z}=0 & \text { on } z=0 .
\end{aligned}
$$

In terms of cylindrical coordinates $(r, \theta, y)$ with associated unit vectors $\mathbf{e}_{r}, \mathbf{e}_{\theta}$ and $\mathbf{e}_{y}$, noting that with the order $\theta, r, y$ the system is right-handed, we obtain from (2.9),

$$
\tilde{\mathbf{u}}=\left(\phi_{r}-\frac{1}{r} \psi_{\theta}+\frac{\mathrm{i} \ell}{k_{2}} \chi_{r}\right) \mathbf{e}_{r}+\left(\frac{1}{r} \phi_{\theta}+\psi_{r}+\frac{\mathrm{i} \ell}{k_{2} r} \chi_{\theta}\right) \mathbf{e}_{\theta}+\left(\mathrm{i} \ell \phi-\frac{q_{2}^{2}}{k_{2}} \chi\right) \mathbf{e}_{y} .
$$

If we want to impose the condition of zero traction on the surface $r=a$ then, since the normal is in the direction of $\mathbf{e}_{r}$, we require the three components $\tau_{r r}, \tau_{\theta r}$ and $\tau_{y r}$ to vanish. Now

$$
\tau_{r r}=-k_{1}^{2} \lambda \phi \mathrm{e}^{\mathrm{i} \ell y}+2 \mu e_{r r}, \quad \tau_{\theta r}=2 \mu e_{\theta r}, \quad \tau_{y r}=2 \mu e_{y r},
$$

where the components of the strain tensor are given by [10, p. 304]

$$
\begin{aligned}
e_{r r} & =u_{r, r}=\mathrm{e}^{\mathrm{i} \ell y}\left(\phi_{r r}+\frac{1}{r^{2}} \psi_{\theta}-\frac{1}{r} \psi_{r \theta}+\frac{\mathrm{i} \ell}{k_{2}} \chi_{r r}\right), \\
e_{y r} & =\frac{\mathrm{e}^{\mathrm{i} \ell y}}{2}\left(2 \mathrm{i} \ell \phi_{r}-\frac{\mathrm{i} \ell}{r} \psi_{\theta}+\frac{\nu^{2}}{k_{2}} \chi_{r}\right), \\
e_{\theta r} & =\frac{\mathrm{e}^{\mathrm{i} \ell y}}{2}\left(\frac{2}{r} \phi_{r \theta}-\frac{1}{r^{2}} \psi_{\theta \theta}+\frac{2 \mathrm{i} \ell}{k_{2} r} \chi_{r \theta}+\psi_{r r}-\frac{2}{r^{2}} \phi_{\theta}-\frac{1}{r} \psi_{r}-\frac{2 \mathrm{i} \ell}{k_{2} r^{2}} \chi_{\theta}\right) .
\end{aligned}
$$

We thus have the boundary conditions

$$
\begin{aligned}
-k_{1}^{2} \lambda \phi+2 \mu\left(\phi_{r r}+\frac{1}{a^{2}} \psi_{\theta}-\frac{1}{a} \psi_{r \theta}+\frac{\mathrm{i} \ell}{k_{2}} \chi_{r r}\right)=0, & \text { on } r=a, \\
2 \mathrm{i} \ell \phi_{r}-\frac{\mathrm{i} \ell}{a} \psi_{\theta}+\frac{\nu^{2}}{k_{2}} \chi_{r}=0, & \text { on } r=a, \\
\frac{2}{a} \phi_{r \theta}-\frac{2}{a^{2}} \phi_{\theta}-\frac{1}{a^{2}} \psi_{\theta \theta}+\psi_{r r}-\frac{1}{a} \psi_{r}+\frac{2 \mathrm{i} \ell}{k_{2} a} \chi_{r \theta}-\frac{2 \mathrm{i} \ell}{k_{2} a^{2}} \chi_{\theta}=0, & \text { on } r=a .
\end{aligned}
$$


In the absence of a cavity, Rayleigh waves can propagate at an angle $\theta$ to the $x$-axis. In this case $\ell=k_{\mathrm{R}} \sin \theta \geq 0$ and $k_{\mathrm{R}}$ is the unique real root of

$$
4 k_{\mathrm{R}}^{2} \beta_{1} \beta_{2}=\left(k_{\mathrm{R}}^{2}+\beta_{2}^{2}\right)^{2}
$$

for which $0<k_{2} / k_{\mathrm{R}}<1$. Here

$$
\beta_{i}=\left(k_{\mathrm{R}}^{2}-k_{i}^{2}\right)^{1 / 2} .
$$

We will explicitly rule out the possibility that Rayleigh waves can propagate in the far field away from the cavity by insisting that $\ell>k_{\mathrm{R}}$. We then have

$$
0<k_{1}<k_{2}<k_{\mathrm{R}}<\ell .
$$

We now expand the wave field in terms of multipoles by writing

$$
\tilde{\mathbf{u}}=\sum_{n, i} \xi_{n}^{(i)} \tilde{\mathbf{u}}_{n}^{(i)},
$$

where $\xi_{n}^{(i)}$ are unknowns to be determined, $\tilde{\mathbf{u}}_{n}^{(i)}$ are multipoles that are defined in Appendix A.1, and $\sum_{n, i}$ is shorthand for $\sum_{n=-\infty}^{\infty} \sum_{i=1}^{3}$. Thus if $\tilde{\mathbf{u}}$ is given by the triple $\{\phi, \psi, \chi\}$ we have

$$
\phi=\sum_{n, i} \mathrm{c}_{i} \xi_{n}^{(i)} \phi_{n}^{(i)}=\sum_{m=-\infty}^{\infty} \mathrm{e}^{\mathrm{i} m \theta}\left(\xi_{m}^{(1)} \mathrm{K}_{m}\left(q_{1} r\right)+\sum_{n, i} \xi_{n}^{(i)} A_{n m}^{(i)} \mathrm{I}_{m}\left(q_{1} r\right)\right),
$$

where $\mathrm{c}_{1}=1, \mathrm{c}_{2}=\mathrm{c}_{3}=\mathrm{i}$ and $\mathrm{I}_{m}(\cdot), \mathrm{K}_{m}(\cdot)$ are modified Bessel functions. Similarly,

$$
\psi=\sum_{n, i} \mathrm{c}_{i} \xi_{n}^{(i)} \psi_{n}^{(i)}=\mathrm{i} \sum_{m=-\infty}^{\infty} \mathrm{e}^{\mathrm{i} m \theta}\left(\xi_{m}^{(2)} \mathrm{K}_{m}\left(q_{2} r\right)+\sum_{n, i} \xi_{n}^{(i)} B_{n m}^{(i)} \mathrm{I}_{m}\left(q_{2} r\right)\right)
$$

and

$$
\chi=\sum_{n, i} \mathrm{c}_{i} \xi_{n}^{(i)} \chi_{n}^{(i)}=\mathrm{i} \sum_{m=-\infty}^{\infty} \mathrm{e}^{\mathrm{i} m \theta}\left(\xi_{m}^{(3)} \mathrm{K}_{m}\left(q_{2} r\right)+\sum_{n, i} \xi_{n}^{(i)} C_{n m}^{(i)} \mathrm{I}_{m}\left(q_{2} r\right)\right) .
$$

The inclusion of the factor $c_{i}$ and the additional factors of $i$ in (2.31) and (2.32) ensure that the system of equations that we obtain for $\xi_{n}^{(i)}$ turns out to be real.

It is now convenient to introduce the notation

$$
\begin{gathered}
I_{n i}=\mathrm{I}_{n}\left(\bar{q}_{i}\right), \quad I_{n i}^{\prime}=\bar{q}_{i} \mathrm{I}_{n}^{\prime}\left(\bar{q}_{i}\right), \quad I_{n i}^{\prime \prime}=\bar{q}_{i}^{2} \mathrm{I}_{n}^{\prime \prime}\left(\bar{q}_{i}\right), \\
K_{n i}=\mathrm{K}_{n}\left(\bar{q}_{i}\right), \quad K_{n i}^{\prime}=\bar{q}_{i} \mathrm{~K}_{n}^{\prime}\left(\bar{q}_{i}\right), \quad K_{n i}^{\prime \prime}=\bar{q}_{i}^{2} \mathrm{~K}_{n}^{\prime \prime}\left(\bar{q}_{i}\right),
\end{gathered}
$$

where an overbar indicates that a quantity has been non-dimensionalised with respect to $a$, and we note that

$$
I_{n i}^{\prime \prime}+I_{n i}^{\prime}-\left(\bar{q}_{i}^{2}+n^{2}\right) I_{n i}=0,
$$

with the equivalent equation for the $K_{n i}$ terms. We also write

$$
\mathcal{I}_{n i}=I_{n i}^{\prime}-I_{n i}, \quad \widetilde{\mathcal{I}}_{n 1}=\left(2 n^{2}-\bar{\nu}^{2}\right) I_{n 1}-2 I_{n 1}^{\prime}, \quad \widetilde{\mathcal{I}}_{n 2}=\left(2 n^{2}+\bar{q}_{2}^{2}\right) I_{n 2}-2 I_{n 2}^{\prime}
$$


with equivalent expressions formed by replacing $\mathcal{I}$ and $I$ with $\mathcal{K}$ and $K$, respectively. We also have

$$
\mu \widetilde{\mathcal{I}}_{m 1}=-\bar{k}_{1}^{2} \lambda I_{m 1}+2 \mu I_{m 1}^{\prime \prime}
$$

which relies on the fact that $k_{1}^{2} \lambda-2 \mu q_{1}^{2}=\mu \nu^{2}$. The boundary condition (2.23) then yields

$$
\begin{aligned}
\widetilde{\mathcal{K}}_{m 1} \xi_{m}^{(1)}+2 m \mathcal{K}_{m 2} \xi_{m}^{(2)} & -\frac{2 \ell}{k_{2}} K_{m 2}^{\prime \prime} \xi_{m}^{(3)} \\
& +\sum_{n, i} \xi_{n}^{(i)}\left[\widetilde{\mathcal{I}}_{m 1} A_{n m}^{(i)}+2 m \mathcal{I}_{m 2} B_{n m}^{(i)}-\frac{2 \ell}{k_{2}} I_{m 2}^{\prime \prime} C_{n m}^{(i)}\right]=0,
\end{aligned}
$$

to be satisfied for each $m \in \mathbb{Z}$ due to orthogonality. Similarly, the boundary condition (2.24) yields

$$
\begin{aligned}
2 \bar{\ell} K_{m 1}^{\prime} \xi_{m}^{(1)}+m \bar{\ell} K_{m 2} \xi_{m}^{(2)}+\frac{\bar{\nu}^{2}}{\bar{k}_{2}} K_{m 2}^{\prime} \xi_{m}^{(3)} & \\
& +\sum_{n, i} \xi_{n}^{(i)}\left[2 \bar{\ell} I_{m 1}^{\prime} A_{n m}^{(i)}+m \bar{\ell} I_{m 2} B_{n m}^{(i)}+\frac{\bar{\nu}^{2}}{\bar{k}_{2}} I_{m 2}^{\prime} C_{n m}^{(i)}\right]=0
\end{aligned}
$$

and the boundary condition (2.25) yields

$$
\begin{aligned}
2 m \mathcal{K}_{m 1} \xi_{m}^{(1)}+\widetilde{\mathcal{K}}_{m 2} & \xi_{m}^{(2)}-\frac{2 m \ell}{k_{2}} \mathcal{K}_{m 2} \xi_{m}^{(3)} \\
& +\sum_{n, i} \xi_{n}^{(i)}\left[2 m \mathcal{I}_{m 1} A_{n m}^{(i)}+\widetilde{\mathcal{I}}_{m 2} B_{n m}^{(i)}-\frac{2 m \ell}{k_{2}} \mathcal{I}_{m 2} C_{n m}^{(i)}\right]=0
\end{aligned}
$$

to be satisfied for each $m \in \mathbb{Z}$ in each case. Equation (2.40) has been simplified using (2.35) to eliminate $I_{m 2}^{\prime \prime}$ and $K_{m 2}^{\prime \prime}$. All the terms in equations (2.38)-(2.40) are real.

3. Symmetry. The geometry we are considering is symmetric about $x=0$, so we can simplify the problem by seeking solutions to (2.38)-(2.40) whose irrotational component, $\phi$, is either symmetric or antisymmetric about $x=0$. Thus, changing $\theta$, $m$ and $n$ to $-\theta,-m$ and $-n$, respectively in (2.30) and then using (A.28) along with $[16, \S 10.27]$, we see that $\phi$ is symmetric if

$$
\xi_{-n}^{(i)}=(-1)^{i+1} \xi_{n}^{(i)} .
$$

Upon making the same transformations in (2.31) and (2.32), it then follows that $\chi$ is also symmetric about $x=0$, whereas $\psi$ is antisymmetric. From equation (2.9) it is clear that if we write $\tilde{\mathbf{u}}(x, z)=u_{1}(x, z) \mathbf{e}_{x}+u_{2}(x, z) \mathbf{e}_{y}+u_{3}(x, z) \mathbf{e}_{z}$ then modes of this type satisfy

$$
\tilde{\mathbf{u}}(-x, z)=-u_{1}(x, z) \mathbf{e}_{x}+u_{2}(x, z) \mathbf{e}_{y}+u_{3}(x, z) \mathbf{e}_{z}
$$

and we have $u_{1}(0, z)=0$. To seek a mode with $\phi$ symmetric about $x=0$, we discard equations with $m<0$ from (2.38)-(2.40) and use (3.1) to eliminate instances of $\xi_{n}^{(i)}$ with $n<0$. We can also deduce that $\xi_{0}^{(2)}=0$ from (3.1), and it then follows from (A.28) that (2.40) is trivially satisfied when $m=0$. 
We can seek for modes with an antisymmetric irrotational component in the same way, except that in this case the factor $(-1)^{i+1}$ in (3.1) must be replaced by $(-1)^{i}$, so that now $\xi_{0}^{(1)}=\xi_{0}^{(3)}=0$, and (2.38) and (2.39) are trivially satisfied when $m=0$. Such modes satisfy

$$
\tilde{\mathbf{u}}(-x, z)=u_{1}(x, z) \mathbf{e}_{x}-u_{2}(x, z) \mathbf{e}_{y}-u_{3}(x, z) \mathbf{e}_{z}
$$

and $u_{2}(0, z)=u_{3}(0, z)=0$.

It is worth noting that, because of the underlying symmetry, the components $\phi$, $\psi$ and $\chi$ are always either real or purely imaginary. Consider a mode in which $\phi$ is symmetric about $x=0$. Then, for the term with $i=1$ in (2.30), we have

$$
\phi^{(1)}=\xi_{0}^{(1)} \phi_{0}^{(1)}+\sum_{n=1}^{\infty} \xi_{n}^{(1)}\left(\phi_{n}^{(1)}+\phi_{-n}^{(1)}\right)
$$

having used (3.1). Now (A.2) gives

$$
\phi_{0}^{(1)}=\mathrm{K}_{0}\left(q_{1} r\right)+\int_{0}^{\infty} A^{(1)} \mathrm{e}^{\gamma_{1}(z-h)} \cos (s x) \frac{\mathrm{d} s}{\gamma_{1}}
$$

because $A^{(1)}$ is an even function, and this is obviously real. Similarly,

$$
\phi_{n}^{(1)}+\phi_{-n}^{(1)}=2 \mathrm{~K}_{n}\left(q_{1} r\right) \cos (n \theta)+\int_{-\infty}^{\infty} A^{(1)} \mathrm{e}^{\gamma_{1}(z-h)}\left(\frac{s-\gamma_{1}}{q_{1}}\right)^{n} \cos (s x) \frac{\mathrm{d} s}{\gamma_{1}},
$$

which is also real. In the same way, we can show that the other terms in (2.30) are real, and using (2.31) and (2.32) we can show that $\psi$ is real, whereas $\chi$ is imaginary (recall that $\xi_{0}^{(2)}=0$ in this case). It then follows from (2.9) that $u_{2}$ is imaginary, whereas the other components of the field are real. This situation is reversed for modes in which $\phi$ is antisymmetric.

4. Unbounded medium. In the case of a cavity in an otherwise uniform elastic medium we could perform exactly the same analysis as in $\S 2$ and the only differences would be that the summations in (2.38)-(2.40) would not be present and as a consequence that the equations for each value of $m$ decouple. Moreover, as Rayleigh waves do not exist in this problem, the less restrictive bound $\ell>k_{2}$ would guarantee that no energy could radiate away from the cylinder. The condition for a wave travelling down the cavity with an $\mathrm{e}^{\mathrm{i} m \theta}$ dependence in this case is simply

$$
\left|\begin{array}{ccc}
\widetilde{\mathcal{K}}_{m 1} & 2 m \mathcal{K}_{m 2} & -\frac{2 \ell}{k_{2}} K_{m 2}^{\prime \prime} \\
2 \bar{\ell} K_{m 1}^{\prime} & m \bar{\ell} K_{m 2} & \frac{\bar{\nu}^{2}}{\bar{k}_{2}} K_{m 2}^{\prime} \\
2 m \mathcal{K}_{m 1} & \widetilde{\mathcal{K}}_{m 2} & -\frac{2 m \ell}{k_{2}} \mathcal{K}_{m 2}
\end{array}\right|=0 .
$$

If we multiply the third column of the determinant by $\bar{k}_{2}$ (which clearly does not change the places at which it vanishes), expand this out and simplify we get

$$
\begin{aligned}
& 4\left(m^{2}-1\right) \bar{k}_{2}^{2} K_{m 1}^{\prime} K_{m 2}^{\prime 2}-2 \bar{\nu}^{4} K_{m 1} K_{m 2}^{\prime 2}+2 \bar{q}_{2}^{2}\left(4 \bar{\ell}^{2}+\bar{k}_{2}^{2}\right) K_{m 1}^{\prime} K_{m 2} K_{m 2}^{\prime} \\
&+2 m^{2} \bar{k}_{2}^{2} \bar{\ell}^{2} K_{m 1} K_{m 2}^{2}+\bar{q}_{2}^{2}\left[\bar{\nu}^{4}+4 m^{2}\left(m^{2}-1-\bar{\nu}^{2}\right)\right] K_{m 1} K_{m 2} K_{m 2}^{\prime} \\
&-4 \bar{\ell}^{2}\left(\bar{q}_{2}^{4}+2 m^{2} \bar{q}_{2}^{2}+m^{4}-m^{2}\right) K_{m 1}^{\prime} K_{m 2}^{2}=0
\end{aligned}
$$


as given in [5] (there is a typographical error in eqn (5) of [5]: the coefficient of $K_{m 1} K_{m 2}^{\prime 2}$ is given as $2 \bar{\nu}^{2}$ rather than $-2 \bar{\nu}^{4}$ ). When $m=0$, corresponding to axisymmetric modes, this reduces to

$$
\begin{aligned}
& \left(\bar{q}_{2} \mathrm{~K}_{0}\left(\bar{q}_{2}\right)+2 \mathrm{~K}_{1}\left(\bar{q}_{2}\right)\right) \\
& \quad \times\left(2 \bar{k}_{2}^{2} \bar{q}_{1} \mathrm{~K}_{1}\left(\bar{q}_{1}\right) \mathrm{K}_{1}\left(\bar{q}_{2}\right)-\bar{\nu}^{4} \mathrm{~K}_{0}\left(\bar{q}_{1}\right) \mathrm{K}_{1}\left(\bar{q}_{2}\right)+4 \bar{\ell}^{2} \bar{q}_{1} \bar{q}_{2} \mathrm{~K}_{1}\left(\bar{q}_{1}\right) \mathrm{K}_{0}\left(\bar{q}_{2}\right)\right)=0,
\end{aligned}
$$

where we have made use of the fact that $K_{0 i}^{\prime}=-\bar{q}_{i} K_{1}\left(\bar{q}_{i}\right)$. The first factor is strictly positive and so we obtain

$$
2 \bar{k}_{2}^{2}-\frac{\bar{\nu}^{4} \mathrm{~K}_{0}\left(\bar{q}_{1}\right)}{\bar{q}_{1} \mathrm{~K}_{1}\left(\bar{q}_{1}\right)}+4 \bar{\ell}^{2} \bar{q}_{2} \frac{\mathrm{K}_{0}\left(\bar{q}_{2}\right)}{\mathrm{K}_{1}\left(\bar{q}_{2}\right)}=0,
$$

which is equivalent to (2.16) of [4].

The authors of [5] found modes for $\ell<k_{\mathrm{R}}$ only. We have checked numerically and our calculations appear to confirm that modes with $\ell>k_{\mathrm{R}}$ do not exist. The significance of $k_{\mathrm{R}}$ in a problem involving only a cylindrical cavity is perhaps not immediately apparent. However, it has long been understood (see, e.g. [20, Chap. I, $\S 4]$ and the references cited therein) that in problems concerned with surface wave propagation along curved surfaces, the waves go over to Rayleigh waves as the radius of curvature tends to infinity (or equivalently, for a fixed geometry, as the frequency tends to infinity) and it is easy to imagine that at high frequencies the cavity wall behaves much like a flat surface along which Rayleigh waves can propagate. This link with Rayleigh waves at high frequencies for propagation along a cylindrical cavity appears to have been first investigated in [15]. If we assume that $\bar{q}_{2}, \bar{\nu}$ and $\bar{\ell}$ are all large then the leading order terms in (4.2) reduce to $\nu^{4}=4 \ell^{2} q_{1} q_{2}$ which is consistent with $\ell \rightarrow k_{\mathrm{R}}$ in this limit (see (2.26)). It should be noted that the difference between $k_{\mathrm{R}}$ and $\ell$ decays relatively slowly as the frequency is increased. For example, with $\sigma=0.2, a=1$ and $k_{2}=50.0$, the mode with $m=0$ (which is closest to the Rayleigh wavenumber), has $\ell \approx 54.7$ whereas $k_{\mathrm{R}} \approx 54.9$ (see also figure 3 in [5]). Consequently, except possibly at extremely high frequencies, modes in the presence of a flat surface and a cavity, which can only exist if $\ell>k_{\mathrm{R}}$, are not closely related to the modes that exist in the presence of a cavity alone.

5. The limit $\ell \rightarrow k_{\mathrm{R}}$. Our numerical results indicate that a different number of trapped modes exist depending on the frequency and the depth of the cavity. In other words, for a given $k_{2}$ and $h$ we find a finite number (possibly zero) of values of $\ell$ for which a non-trivial solution to (2.38)-(2.40) exists. Modes cut on and off in the limit $\ell \rightarrow k_{\mathrm{R}}$ and due to the singular nature of some of the multipoles, numerical computations can become problematic. Hence it is helpful to determine the cut-off values by first analysing the equations in this limit.

Any solution has a Helmholtz decomposition $\{\phi, \psi, \chi\}$ and from (2.30)-(2.32) together with the results in Appendix A.3 we can see that $\phi$ and $\chi$ contain singular terms in the limit $\ell \rightarrow k_{\mathrm{R}}$ but $\psi$ does not. It is clear from (2.9) that, since all three components of $\tilde{\mathbf{u}}$ must remain bounded, each of $\phi$ and $\chi$ needs to be regular in order for the triple to represent a physical solution and this imposes conditions on the coefficients $\xi_{n}^{(i)}$. To see this we write $\ell^{2}=k_{\mathrm{R}}^{2}+\epsilon^{2}, \epsilon>0$, and let

$$
\xi_{n}^{(i)}=x_{n}^{(i)}+\epsilon y_{n}^{(i)}+O\left(\epsilon^{2}\right) .
$$

After substituting this into (2.30) and using (A.37) and (A.40) (or alternatively by using (A.46), (A.49) and (A.52) in (2.30)), we can deduce that the singular terms will 
cancel if

$$
\sum_{n=-\infty}^{\infty}(-1)^{n}\left(x_{n}^{(1)}+S x_{n}^{(3)}\right)=0
$$

where

$$
S=-k_{2}^{-1} \sqrt{\beta_{1} \beta_{2}} \mathrm{e}^{\left(\beta_{1}-\beta_{2}\right) h} .
$$

Consideration of $\chi$ leads to exactly the same condition, in view of (A.45).

For modes which are $\phi$-antisymmetric, equation (3.1) shows that $x_{n}^{(1)}=-x_{-n}^{(1)}$ and likewise for $x_{n}^{(3)}$. Hence in this case the singularities automatically cancel and the cut-off values can be computed by discarding the singular terms and setting $\epsilon=0$. For $\phi$-symmetric modes the situation is more complicated. If (5.2) is satisfied, we have

$$
\sum_{n, i} \xi_{n}^{(i)} A_{n m}^{(i)}=(-1)^{m} a^{(1)} Y+\sum_{n, i} x_{n}^{(i)} \operatorname{Re} \widehat{A}_{n m}^{(i)}+O(\epsilon)
$$

where $a^{(1)}$ and $\widehat{A}_{n m}^{(i)}$ are defined in Appendix A.3 and

$$
Y=\sum_{n=-\infty}^{\infty}(-1)^{n}\left(y_{n}^{(1)}+S y_{n}^{(3)}\right) \text {. }
$$

Similarly,

$$
\sum_{n, i} \xi_{n}^{(i)} C_{n m}^{(i)}=(-1)^{m} c^{(1)} Y+\sum_{n, i} x_{n}^{(i)} \operatorname{Re} \widehat{C}_{n m}^{(i)}+O(\epsilon) .
$$

Thus in the limit as $\ell \rightarrow k_{\mathrm{R}}$ we can introduce an extra unknown, $Y$, and an extra equation, (5.2), expand (2.38)-(2.40) about $\epsilon=0$ and then set $\epsilon=0$. For example, (2.38) becomes

$$
\begin{aligned}
\widetilde{\mathcal{K}}_{m 1} x_{m}^{(1)}+2 m \mathcal{K}_{m 2} x_{m}^{(2)}-\frac{2 \ell}{k_{2}} K_{m 2}^{\prime \prime} x_{m}^{(3)}+(-1)^{m}\left(\widetilde{\mathcal{I}}_{m 1} a^{(1)}-\frac{2 \ell}{k_{2}} I_{m 2}^{\prime \prime} c^{(1)}\right) Y \\
+\sum_{n, i} x_{n}^{(i)} \operatorname{Re}\left[\widetilde{\mathcal{I}}_{m 1} \widehat{A}_{n m}^{(i)}+2 m \mathcal{I}_{m 2} \widehat{B}_{n m}^{(i)}-\frac{2 \ell}{k_{2}} I_{m 2}^{\prime \prime} \widehat{C}_{n m}^{(i)}\right]=0 .
\end{aligned}
$$

6. Nature of the field. In our formulation, the displacement is expressed as a series of multipoles and we can gain some insight into the nature of the field, and in particular where it is localised, by analysing the behaviour of the multipoles in certain limits. For the terms outside the integrals in (A.2), (A.7) and (A.12), we can use the formula $[16,10.25 .3]$

$$
\mathrm{K}_{n}\left(q_{j} r\right) \sim \sqrt{\frac{\pi}{2 q_{j} r}} \mathrm{e}^{-q_{j} r}, \quad \text { as } \quad q_{j} r \rightarrow \infty .
$$

Thus, contributions from these terms are localised about the centre of the cavity, an effect which becomes more profound as the frequency is increased.

In determining the behaviour of the integrals themselves, we may assume that $x>0$, since results for $x<0$ can always be obtained by symmetry. First consider 
(A.2). We begin by shifting the entire path of integration into the upper half of the complex $s$ plane, without moving close to the branch point at $s=\mathrm{i} q_{1}$. (To determine the precise behaviour we would need to deform the contour into the steepest descents path that passes through the saddle point at $s=\mathrm{i} q_{1} x / \sqrt{x^{2}+(h-z)^{2}}$ but simply raising the path of integration is sufficient to show that the integrals decay exponentially in both $x$ and $z$.) This shows that the contribution from the integral is localised in the region between the cavity and the flat free surface (i.e. the part of the domain $z<0$ closest to the image point at $(x, z)=(0, h)$ ), and is exponentially small elsewhere. Again, this effect is more profound at higher frequencies. At low frequencies the branch point at $s=\mathrm{i} q_{1}$ forces part of the contour to remain close to the origin, reducing the rate of exponential decay in $x$, whilst proximity to the branch point itself reduces the rate of exponential decay in $z$. If the shifted contour passes above the pole at $s=\mathrm{i} \sqrt{\ell^{2}-k_{\mathrm{R}}^{2}}$, then a residue contribution needs to be included. In cases where $\ell$ is significantly greater than $k_{\mathrm{R}}$, this contribution is also localised in the region between the cavity and the free surface. On the other hand, as $\ell \rightarrow k_{\mathrm{R}}$, the pole moves close to the origin, and the exponential decay in $x$ is weakened.

We can also examine the nature of the field in the limit as $\ell \rightarrow k_{R}$. From (2.30), along with (A.46), (A.49) and (A.52), we have

$$
\phi=\phi_{\mathrm{res}}+\sum_{n, i} \mathrm{c}_{i} \xi_{n}^{(i)} \widehat{\phi}_{n}^{(i)},
$$

where the contribution from the residues is given by

$$
\begin{array}{r}
\phi_{\text {res }}=\frac{2 \pi}{\epsilon p_{0}} k_{\mathrm{R}}^{2} \beta_{2} \mathrm{e}^{\beta_{1}(z-h)} \sum_{n=-\infty}^{\infty}(-1)^{n}\left[\xi_{n}^{(1)}\left(1-x \epsilon-\frac{\mathrm{i} n \epsilon}{\beta_{1}}\right)-\mathrm{i} \epsilon \xi_{n}^{(2)} \frac{k_{2} S}{k_{\mathrm{R}} \beta_{2}}\right. \\
\left.+S \xi_{n}^{(3)}\left(1-x \epsilon-\frac{\mathrm{i} n \epsilon}{\beta_{2}}\right)\right]+O(\epsilon),
\end{array}
$$

for fixed, positive $x$. Here, $S$ is defined in (5.3), and $p_{0}$ is given by (A.33).

For the case of a $\phi$-symmetric mode we have, from (3.1),

$$
\sum_{n=-\infty}^{\infty}( \pm 1)^{n} n \xi_{n}^{(1)}=\sum_{n=-\infty}^{\infty}( \pm 1)^{n} \xi_{n}^{(2)}=\sum_{n=-\infty}^{\infty}( \pm 1)^{n} n \xi_{n}^{(3)}=0
$$

and hence

$$
\phi_{\text {res }}=\frac{2 \pi}{\epsilon p_{0}} k_{\mathrm{R}}^{2} \beta_{2} \mathrm{e}^{\beta_{1}(z-h)}(1-x \epsilon) \sum_{n=-\infty}^{\infty}(-1)^{n}\left(\xi_{n}^{(1)}+S \xi_{n}^{(3)}\right)+O(\epsilon) .
$$

Since (5.2) applies for any mode, this reduces to

$$
\phi_{\text {res }}=\frac{2 \pi Y}{p_{0}} k_{\mathrm{R}}^{2} \beta_{2} \mathrm{e}^{\beta_{1}(z-h)}+O(\epsilon),
$$

having used (5.1) and (5.5). Following the same procedure for the other terms, we find that $\psi_{\text {res }}=O(\epsilon)$, and

$$
\chi_{\mathrm{res}}=\frac{2 \pi \mathrm{i} Y}{p_{0}} k_{\mathrm{R}}^{2} k_{2} \beta_{1}^{1 / 2} \beta_{2}^{-1 / 2} \mathrm{e}^{\beta_{2} z-\beta_{1} h}+O(\epsilon) .
$$


Comparing these to equations (3.8) and (3.9) in [13] with $\theta_{\text {inc }}=\pi / 2$ (noting that $\kappa_{i}^{2}=-\beta_{i}^{2}$ when $\ell=k_{\mathrm{R}}$ ), we see that the $O(1)$ terms constitute a Rayleigh wave propagating in the $y$-direction.

For the case of a $\phi$-antisymmetric mode, a very similar analysis shows that the $O(1)$ contribution from the residues is again a Rayleigh wave propagating in the $y$ direction. Of course for $x<0$ we get a Rayleigh wave with a different complex amplitude coefficient (since $\phi$ and $\chi$ are antisymmetric in $x$ ) with the apparent discontinuity at $x=0$ smoothed out by the $\widehat{\phi}_{n}^{(i)}$ and $\widehat{\chi}_{n}^{(i)}$ terms.

On the basis of this analysis, we can make some predictions about the parameter regimes in which we should expect to find trapped modes. First, consider the cavity depth $h$. Since the exponential factors in the multipole integrals have the form $\mathrm{e}^{-2 \gamma_{i} h}$ or $\mathrm{e}^{-\left(\gamma_{1}+\gamma_{2}\right) h}$, where $\gamma_{i}^{2}=s^{2}+\ell^{2}-k_{i}^{2}$ (see Appendix A.1), it follows that increasing $h$ weakens the coupling between the flat surface and the cavity. A $\phi$-symmetric mode can persist in such cases, as a small perturbation of a Rayleigh wave, but a $\phi$-antisymmetric mode cannot (because other contributions are needed to smooth the transition between different amplitudes for $x>0$ and $x<0$ ). Therefore we should not expect to find $\phi$-antisymmetric modes at large depths. Similarly, increasing the frequency causes the difference $k_{\mathrm{R}}-k_{i}$ (the lower limit for $\ell-k_{i}$ ) to increase, but this is equivalent to increasing both the cavity depth $h$ and the radius $a$, so the weakening of the coupling between the cavity and the flat surface is relatively slow to take effect. Therefore we should expect that $\phi$-symmetric modes can persist at high frequencies, but that $\phi$-antisymmetric modes will eventually cut off. Finally, we observe that increasing $\ell$ weakens the coupling and increases the distinction between the trapped modes and the Rayleigh wave. Therefore we cannot expect to find modes with $\ell \gg k_{\mathrm{R}}$, and should only expect to find a significant difference between $\ell$ and $k_{\mathrm{R}}$ in cases where the coupling between the cavity and the flat surface is particularly strong.

7. Numerical Results. The first step in locating solutions to (2.38)-(2.40) is to evaluate the integral representations for $A_{n m}^{(i)}, B_{n m}^{(i)}$ and $C_{n m}^{(i)}$ given by (A.23), (A.25) and (A.27), respectively. For most parameters, the composite trapezium rule yields very accurate results. If contributions from $|s|>s_{\max }$ are negligible, then the error is approximately proportional to $\mathrm{e}^{-\pi N d / s_{\max }}$, where $N$ is the number of trapeziums used and $d$ is the perpendicular distance from the path of integration to the nearest singularity in the $s$ plane [17]. In some cases, this distance was increased by raising the path of integration so that it passed between the pole at $s=\mathrm{i} \sqrt{\ell^{2}-k_{\mathrm{R}}^{2}}$ and the branch point at $s=\mathrm{i} q_{2}$. In cases where no path on which $d \geq 1$ exists, an adaptive Gaussian quadrature scheme was used instead.

Next, we truncate the multipole expansions in (2.30)-(2.32), retaining terms up to $|m|=|n|=T$. Separate homogeneous linear systems for the coefficients $\xi_{n}^{(i)}$ corresponding to $\phi$-symmetric and $\phi$-antisymmetric modes can then be formed by combining the truncated forms of (2.38)-(2.40), taking into account the results of $\S 3$ to eliminate equations with $m<0$ and one or two equations with $m=0$. Each mode then corresponds to a value for $\ell$ which results in a zero determinant. Care is needed in calculating the determinants, because some row orderings (such as grouping the equations that come from each of (2.38), (2.39) and (2.40) together) lead to numerical issues. We have found that placing equations with $m=0$ at the top of the matrices, followed by those with $m=1, m=2$, etc. and then using Gaussian elimination with partial pivoting is an effective strategy.

In what follows, $\sigma$ (Poisson's ratio) is fixed at 0.2 , so that $\Lambda=0.375$ (see (2.4)). 


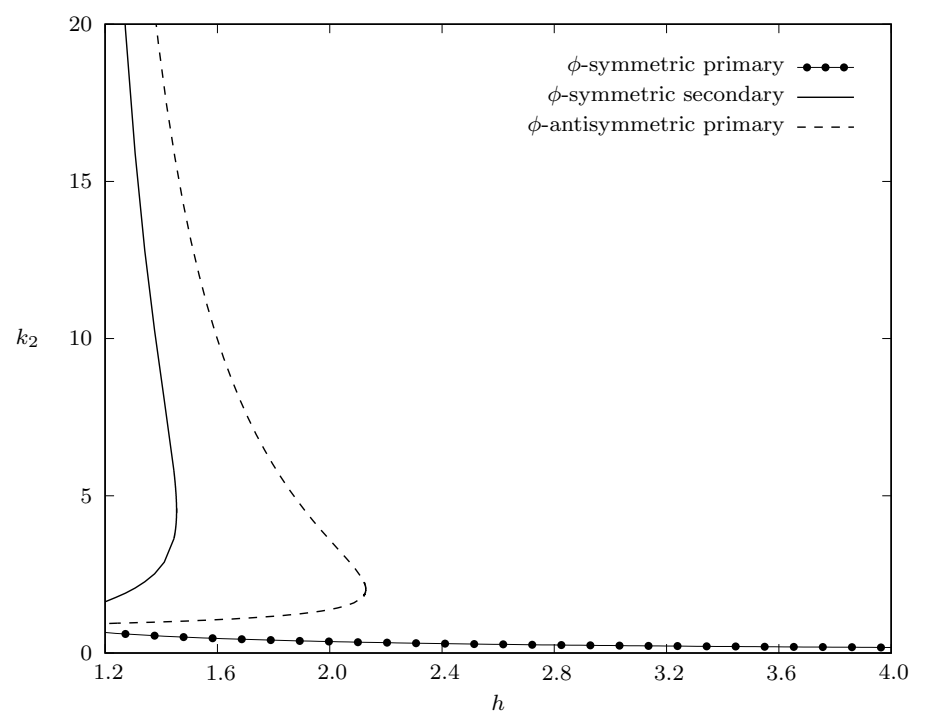

FIG. 1. Critical wavenumbers at which different trapped modes cut on and cut off.

This value is representative of many rocks $[9,8]$. The ratio $k_{2} / k_{\mathrm{R}}$ is then fixed at approximately 0.911 by (A.17). We also fix the cavity radius at unity. The value used for $T$ was varied up to a maximum of 30 to account for different frequencies and cavity depths. In general, the cavity depth has a far stronger effect on convergence than frequency. All of the results presented were obtained using a Fortran 2003 code running at double precision, and were confirmed by recalculating with a larger value of $T$.

Figure 1 shows the frequencies at which different modes cut on and off as $k_{2}$ increases, for varying cavity depths $h$. These results were obtained by seeking solutions with $\ell=k_{\mathrm{R}}$, as described in $\S 5$. Whilst it is possible to obtain similar results using only the system formed from (2.38)-(2.40) with $\ell$ close to $k_{\mathrm{R}}$, it is difficult to guarantee accuracy using this approach. We find that a $\phi$-symmetric mode exists at all depths, for frequencies above the dashed line in the figure. Henceforth we will refer to this as the primary $\phi$-symmetric mode. The presence of a cut-on frequency below which no modes exist, which contrasts with the equivalent water-wave problem where modes exist for all frequencies, is noteworthy and has been observed previously in a related problem [7].

Attempts to locate cut-off frequencies for the primary $\phi$-symmetric mode (including calculations at quadruple precision, using very high truncations and frequencies) have been unsuccessful. Instead, our results indicate that, in this case, $\ell \rightarrow k_{\mathrm{R}}$ as $k_{2} \rightarrow \infty$. Other modes exist only for shallow cavity depths. For $h \lesssim 2.13$, there is a mode with $\phi$ antisymmetric, and for $h \lesssim 1.45$ a secondary $\phi$-symmetric mode appears. In contrast to the primary $\phi$-symmetric mode, these exist within a finite range of frequencies between a cut-on and a cut-off, for parameter sets to the left of the grey and solid black curves, respectively. In both cases, the cut-off frequency increases rapidly as the cavity depth is decreased. Additional modes, both $\phi$-symmetric and $\phi$-antisymmetric, exist at very shallow depths, and are not shown in the figure.

Figure 2 shows dispersion curves which illustrate how the axial wavenumber $\ell$ varies with frequency, for $\phi$-symmetric modes at four different cavity depths. Rather 


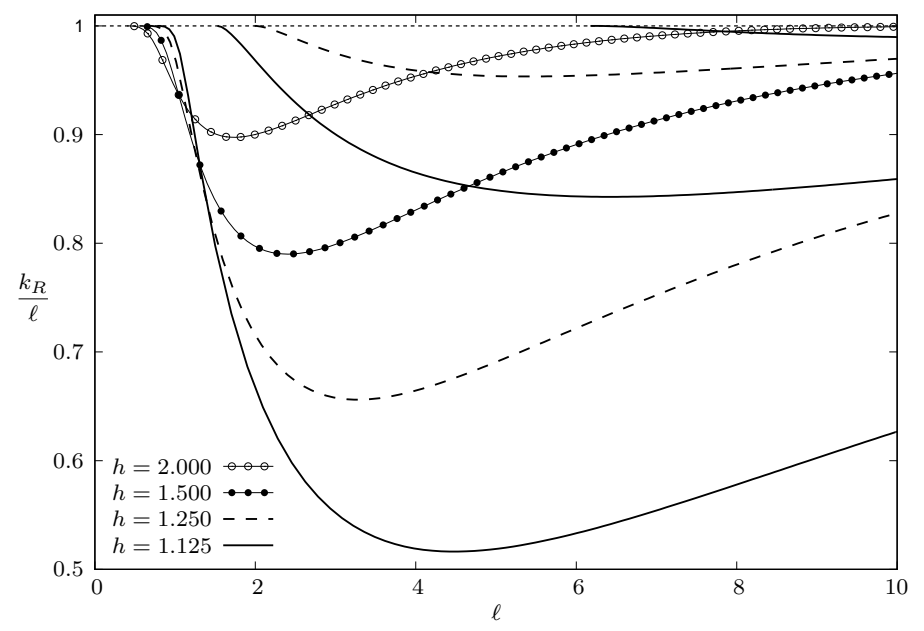

FIG. 2. Axial wavenumbers plotted against axial wave speed (non-dimensionalised with respect to the Rayleigh wave speed) for $\phi$-symmetric trapped modes at various depths. A single mode exists for $h=2$ and $h=1.5$, two modes for $h=1.25$ and three for $h=1.125$.

than plot $\ell$ versus $k_{2}$, which results in curves very close to the line $\ell=k_{\mathrm{R}}$ that are hard to distinguish, we instead choose $k_{\mathrm{R}} / \ell$ as the ordinate. This is the speed of the trapped mode along the cylinder, non-dimensionalised with respect to the Rayleigh wave speed. As $\ell$ increases along each curve, so does the frequency. For each mode, as the frequency increases above the cut-on, the axial wavenumber first moves away from the Rayleigh wavenumber, but the difference eventually starts to decrease. The mode disappears if another solution with $\ell=k_{\mathrm{R}}$ is reached. In agreement with the results in figure 1, there is a single $\phi$-symmetric mode for $h=2$ and $h=1.5$, but the secondary mode is visible for $h=1.25$ and $h=1.125$. These cut on at $k_{2} \approx 1.81$ and $k_{2} \approx 1.39$, respectively. A tertiary mode, with $\ell$ very close to the Rayleigh wavenumber, was also found for $h=1.125$; this cuts on at $k_{2} \approx 5.63$. For shallow depths, the secondary and tertiary modes persist up to very high frequencies. For example, when $h=1.25$ the secondary mode cuts off at $k_{2} \approx 23.1$, at which point we have $k_{\mathrm{R}}=\ell \approx 25.3$. Figure 3 shows the dispersion curves for $\phi$-antisymmetric modes, at the same cavity depths. In this case, there is only one mode for $h=2, h=1.5$ and $h=1.25$, but a secondary mode is visible for $h=1.125$, cutting on at $k_{2} \approx 2.67$.

Contour plots showing the magnitude of the field $\tilde{\mathbf{u}}$ (defined in (2.9)) are presented in figures $4-7$, with scaling applied so that $0 \leq|\tilde{\mathbf{u}}| \leq 1$. Darker regions correspond to larger values. In figure 4 , the cavity is placed at a relatively large depth $(h=4)$, so that only the primary $\phi$-symmetric mode exists. At low frequency (figure 4(a)), the effect of the mode is noticeable far below the cavity, but is clearly at its strongest near the flat free surface. As the frequency is increased (figures 4(b)-(c)), the mode gradually becomes more localised at the flat surface, eventually evolving into a small perturbation of a Rayleigh wave. In the last image, we have $k_{\mathrm{R}}=\ell$ to four significant figures.

Figure 5 again shows the primary $\phi$-symmetric mode, this time for a more moderate cavity depth $(h=2)$. At low frequency, the pattern is similar to the $h=4$ case, though the rate of decay in $z$ is clearly greater. In figure $5(\mathrm{~b})$, the frequency has been increased to $k_{2}=5.0$, so that $\ell-k_{\mathrm{R}} \approx 0.116$, which is close to the maximum difference for these parameters (see figure 2). The mode is localised in the region 


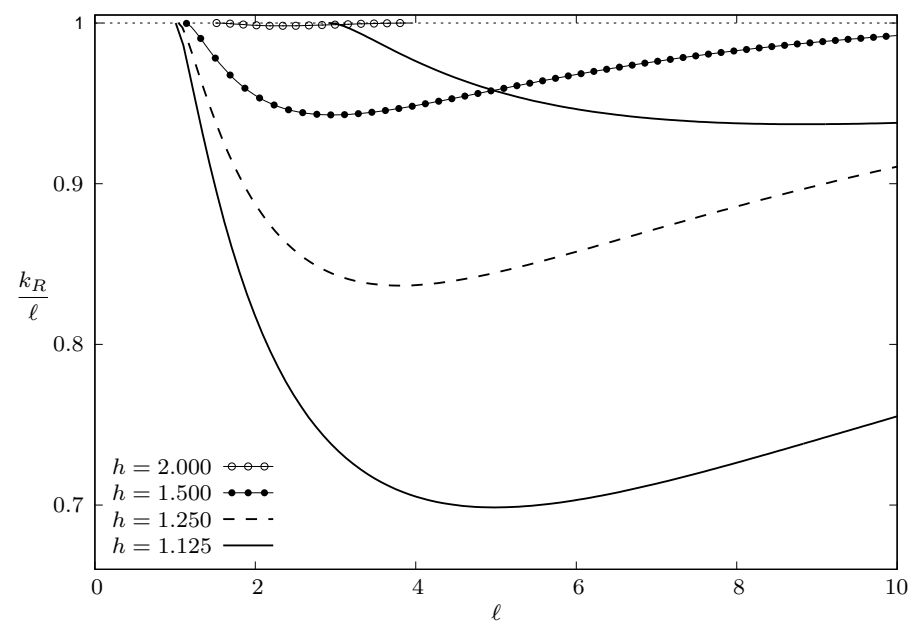

FIG. 3. Axial wavenumbers plotted against axial wave speed (non-dimensionalised with respect to the Rayleigh wave speed) for $\phi$-antisymmetric trapped modes at various depths. A single mode exists for $h=2, h=1.5$ and $h=1.25$, with two modes for $h=1.125$.

(a)

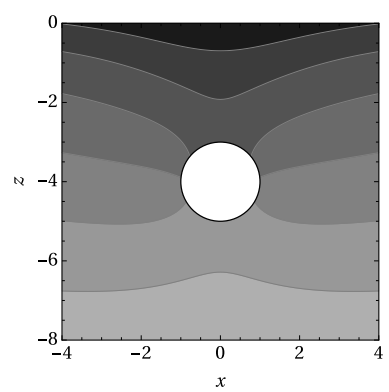

(b)

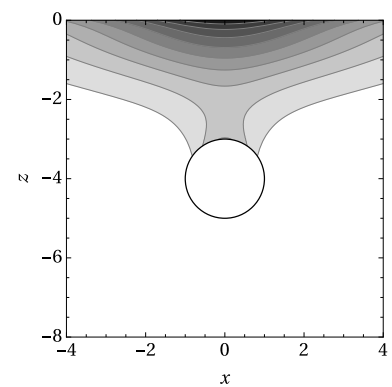

(c)

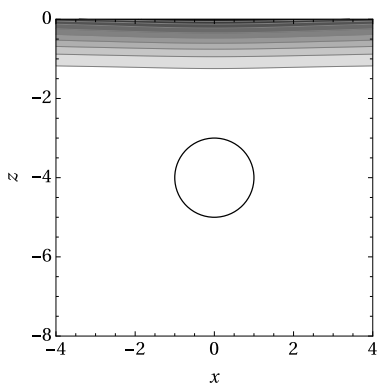

FIG. 4. Contour plots showing $|\tilde{\mathbf{u}}|$ for the primary $\phi$-symmetric mode at depth $h=4$. (a) $k_{2}=0.25$, (b) $k_{2}=1.7$, (c) $k_{2}=3.2$. The mode exists for $k_{2} \gtrsim 0.177$.

between the cavity and the flat free surface. In figure $5(\mathrm{c})$, the frequency has been further increased so that now $k_{2}=10.0$, and we have $\ell=k_{\mathrm{R}}$ to four significant figures. As in figure 4(c), the mode is now a small perturbation of a Rayleigh wave, but the disturbance near $x=0$, due to coupling with the cavity, is much stronger.

The characteristics of the image shown in $5(\mathrm{~b})$ are common to all cases where the frequency is relatively high, but the difference between $\ell$ and $k_{\mathrm{R}}$ is appreciable (so that the mode is not similar to a Rayleigh wave). Heuristically, this can be understood as follows. If an observer is located on the surface of the cavity, then the effects of the singularities at $(x, z)=(0, h)$ and $(x, z)=(0,-h)$ must be similar in magnitude, so that the boundary conditions at $r=a$ can be satisfied. Moving away from the cavity boundary in any direction (while remaining inside the domain of the problem) will cause the contribution from $(0,-h)$ to decrease exponentially, according to (6.1). However, moving into the region between the cavity and the flat surface brings the observer closer to the image point at $(0, h)$, so that the strength of this contribution 
(a)

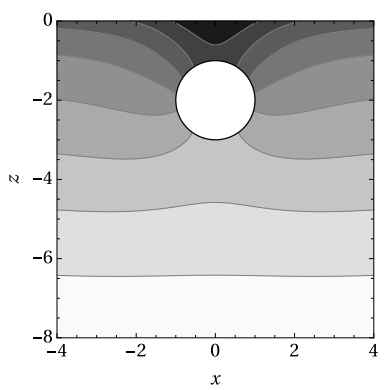

(b)

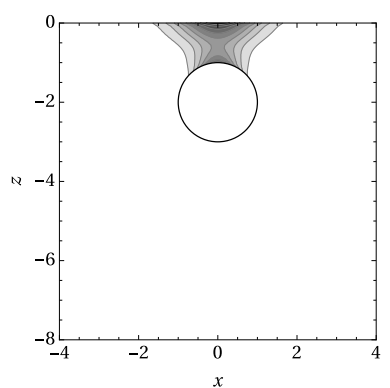

(c)

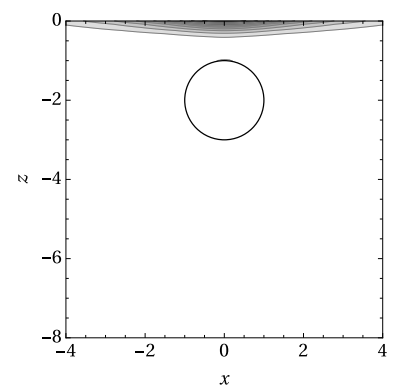

FIG. 5. Contour plots showing $|\tilde{\mathbf{u}}|$ for the primary symmetric mode at depth $h=2$. (a) $k_{2}=0.4$, (b) $k_{2}=5.0$, (c) $k_{2}=10.0$. The mode exists for $k_{2} \gtrsim 0.364$.

(a)

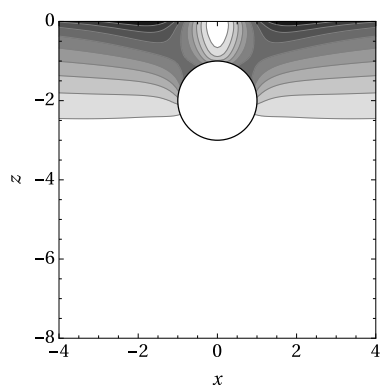

(b)

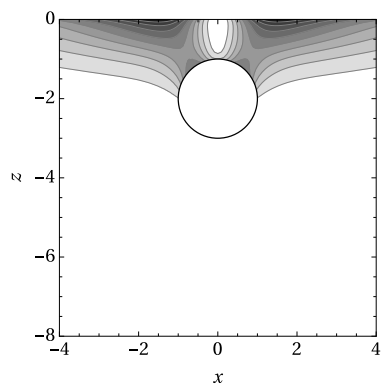

(c)

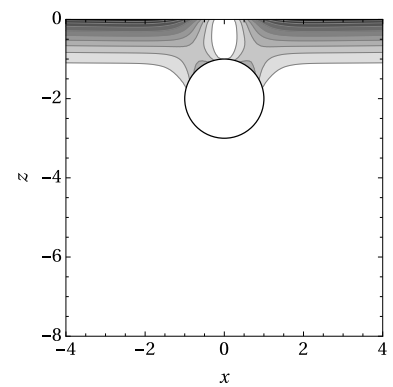

FIG. 6. Contour plots showing $|\tilde{\mathbf{u}}|$ for the $\phi$-antisymmetric mode at depth $h=2$. (a) $k_{2}=1.45$, (b) $k_{2}=2.5$, (c) $k_{2}=3.55$. The mode exists for $1.38 \lesssim k_{2} \lesssim 3.60$.

increases exponentially.

Figure 6 shows the $\phi$-antisymmetric mode at cavity depth $h=2.0$. This mode does not exist at very low frequencies (see figure 1), so its effect is generally very weak below the cavity. Similarity to the Rayleigh wave is evident in all three cases, except near $x=0$, where the transitional region which accounts for the change in complex amplitude across $x=0$ is clearly evident. This is to be expected, since $\ell$ is always close the Rayleigh wavenumber (see figure 3). The resemblance is greatest when the frequency is closest to the cut-off (figure 6(c)), since other contributions to the field are then strongly localised in the region between the cavity and the flat surface.

In figure 7 , the depth and frequency are fixed at $h=1.25$ and $k_{2}=2.0$ and the plots show the three possible modes that can exist. These are the primary and secondary $\phi$-symmetric modes, along with the $\phi$-antisymmetric mode. In this case, the Rayleigh wavenumber is approximately 2.20 and the respective values for $\ell$ are approximately $3.35,2.20$ and 2.57 . Consequently, the primary $\phi$-symmetric and the $\phi$-antisymmetric mode are localised in the region between the cavity and the flat surface, but the effect of the secondary $\phi$-symmetric mode is visible for a much wider range of $x$ values. The curves in figure 2 enable us to predict how the pattern of the secondary $\phi$-symmetric mode evolves as the frequency is increased (not shown in the 

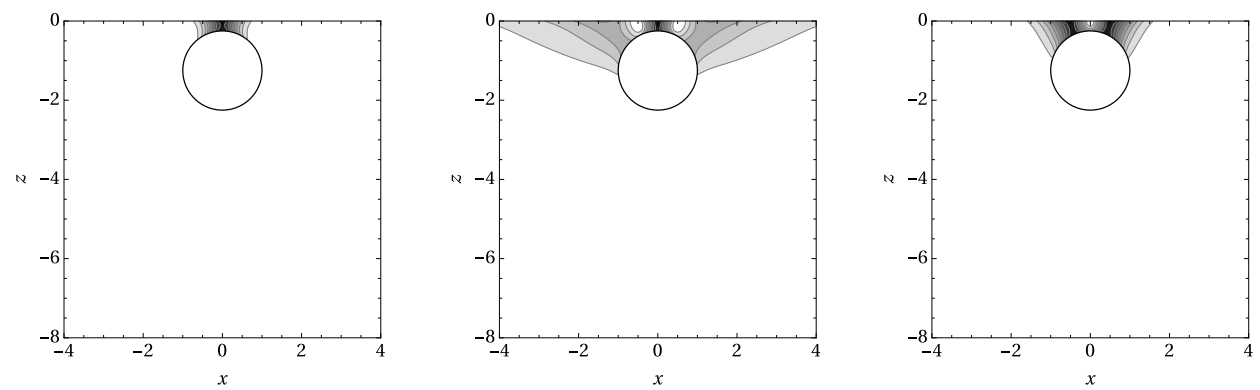

FIG. 7. Contour plots showing $|\tilde{\mathbf{u}}|$ for the different modes at depth $h=1.25$ and $k_{2}=2.0$. (a) primary $\phi$-symmetric mode, (b) secondary $\phi$-symmetric mode, (c) $\phi$-antisymmetric mode.

contour plots). Initially, the range of $x$ values for which its effect is visible is reduced, so that it becomes localised in the region between the cavity and the flat surface. As $\ell \rightarrow k_{\mathrm{R}}$, its effect widens again, but it does not attain a form similar to a Rayleigh wave, because the depth is very shallow, meaning that other effects are always strong in the region close to $x=0$.

8. Conclusion. Using a method based on multipole expansions, we have demonstrated the existence of trapped elastic waves above a circular cylindrical cavity in a half-space. These modes propagate parallel to the cylinder and their axial wavenumber $\ell$ exceeds the Rayleigh wavenumber $k_{\mathrm{R}}$, so that the field decays exponentially in other directions. The modes can be categorised by whether the irrotational part of the field is symmetric or antisymmetric. We have obtained dispersion relations connecting the frequency with the axial wavenumber for modes of both types, and solved these relations numerically. We have also determined the points in parameter space at which the modes cut on and off, and presented contour plots showing the associated displacement fields to illustrate how the modes can be localised in the vicinity of the cavity and the flat free surface.

The modes we have studied are analogous to trapped water waves which propagate above a rigid circular cylinder submerged in infinitely deep water. In that case, a single scalar field serves to define the solution, whereas in this case three coupled functions are required. In the water wave problem, at least one mode exists for all depths and frequencies. However, in our case we have found that for each submergence depth there is a cut-on frequency below which no trapped modes exist, though there is no upper cut-off. In both cases, the number of different modes that can exist increases as the submergence depth is reduced. For deep cavities, at most one mode can exist and this takes the form of a small perturbation of a Rayleigh wave. In the water wave problem, a deeply submerged cylinder always supports a single mode, which is a small perturbation of a plane wave. Another difference between the two problems is the existence of modes propagating along a cylindrical cavity in an otherwise unbounded medium (with $\ell<k_{\mathrm{R}}$ ). No such modes exist in the analogous water wave problem.

\section{Appendix A. Mutipoles.}

A.1. Construction. The aim is to construct solutions to Navier's equation (2.2) which are (i) of the form $\mathbf{u}=\tilde{\mathbf{u}}(x, z) \mathrm{e}^{\mathrm{i} \ell y}$, (ii) singular at $z=-h(h>0)$, (iii) satisfy 
the condition of zero traction on $z=0$ and (iv) decay as $|x| \rightarrow \infty$. The construction follows closely that in [13] though different scalings are used here. From [13, eqn (A.2)] we have, with $q>0$,
(A.1)
$\mathrm{K}_{n}(q r) \mathrm{e}^{\mathrm{i} n \theta}=\frac{1}{2 q^{n \operatorname{sgn}(z+h)}} \int_{-\infty}^{\infty} \mathrm{e}^{-\tilde{\gamma}(s ; \mathrm{i} q)|z+h|} \mathrm{e}^{\mathrm{i} s x}(s-\tilde{\gamma}(s ; \mathrm{i} q))^{n \operatorname{sgn}(z+h)} \frac{\mathrm{d} s}{\tilde{\gamma}(s ; \mathrm{i} q)}$,

where $\tilde{\gamma}(s ; k)=\left(s^{2}-k^{2}\right)^{1 / 2}$ and $\operatorname{Re} \tilde{\gamma}>0$ as $|s| \rightarrow \infty$.

Multipoles $\tilde{\mathbf{u}}_{n}^{(i)}$ are triples $\left\{\phi_{n}^{(i)}, \psi_{n}^{(i)}, \chi_{n}^{(i)}\right\}, i=1,2,3$ of the form

$$
\begin{aligned}
\phi_{n}^{(1)} & =\mathrm{K}_{n}\left(q_{1} r\right) \mathrm{e}^{\mathrm{i} n \theta}+\frac{1}{2 q_{1}^{n}} \int_{-\infty}^{\infty} A^{(1)} \mathrm{e}^{\gamma_{1}(z-h)} \mathrm{e}^{\mathrm{i} s x}\left(s-\gamma_{1}\right)^{n} \frac{\mathrm{d} s}{\gamma_{1}}, \\
& =\frac{1}{2 q_{1}^{n}} \int_{-\infty}^{\infty}\left[\mathrm{e}^{-\gamma_{1}(z+h)}+A^{(1)} \mathrm{e}^{\gamma_{1}(z-h)}\right] \mathrm{e}^{\mathrm{i} s x}\left(s-\gamma_{1}\right)^{n} \frac{\mathrm{d} s}{\gamma_{1}}, \quad z>-h, \\
\psi_{n}^{(1)} & =\frac{\mathrm{i}}{2 q_{1}^{n}} \int_{-\infty}^{\infty} B^{(1)} \mathrm{e}^{-\gamma_{1} h} \mathrm{e}^{\gamma_{2} z} \mathrm{e}^{\mathrm{i} s x}\left(s-\gamma_{1}\right)^{n} \mathrm{~d} s, \\
\chi_{n}^{(1)} & =\frac{\mathrm{i}}{2 q_{1}^{n}} \int_{-\infty}^{\infty} C^{(1)} \mathrm{e}^{-\gamma_{1} h} \mathrm{e}^{\gamma_{2} z} \mathrm{e}^{\mathrm{i} s x}\left(s-\gamma_{1}\right)^{n} \mathrm{~d} s,
\end{aligned}
$$

and we have

$$
\begin{aligned}
\phi_{n}^{(2)} & =\frac{-\mathrm{i}}{2 q_{2}^{n}} \int_{-\infty}^{\infty} A^{(2)} \mathrm{e}^{-\gamma_{2} h} \mathrm{e}^{\gamma_{1} z} \mathrm{e}^{\mathrm{i} s x}\left(s-\gamma_{2}\right)^{n} \mathrm{~d} s, \\
\psi_{n}^{(2)} & =\mathrm{K}_{n}\left(q_{2} r\right) \mathrm{e}^{\mathrm{i} i \theta}+\frac{1}{2 q_{2}^{n}} \int_{-\infty}^{\infty} B^{(2)} \mathrm{e}^{\gamma_{2}(z-h)} \mathrm{e}^{\mathrm{i} s x}\left(s-\gamma_{2}\right)^{n} \frac{\mathrm{d} s}{\gamma_{2}}, \\
& =\frac{1}{2 q_{2}^{n}} \int_{-\infty}^{\infty}\left[\mathrm{e}^{-\gamma_{2}(z+h)}+B^{(2)} \mathrm{e}^{\gamma_{2}(z-h)}\right] \mathrm{e}^{\mathrm{i} s x}\left(s-\gamma_{2}\right)^{n} \frac{\mathrm{d} s}{\gamma_{2}}, \quad z>-h, \\
\chi_{n}^{(2)} & =\frac{1}{2 q_{2}^{n}} \int_{-\infty}^{\infty} C^{(2)} \mathrm{e}^{\gamma_{2}(z-h)} \mathrm{e}^{\mathrm{i} s x}\left(s-\gamma_{2}\right)^{n} \mathrm{~d} s,
\end{aligned}
$$

and

$$
\phi_{n}^{(3)}=\frac{-\mathrm{i}}{2 q_{2}^{n}} \int_{-\infty}^{\infty} A^{(3)} \mathrm{e}^{-\gamma_{2} h} \mathrm{e}^{\gamma_{1} z} \mathrm{e}^{\mathrm{i} s x}\left(s-\gamma_{2}\right)^{n} \mathrm{~d} s,
$$

$$
\begin{aligned}
& \psi_{n}^{(3)}=\frac{1}{2 q_{2}^{n}} \int_{-\infty}^{\infty} B^{(3)} \mathrm{e}^{\gamma_{2}(z-h)} \mathrm{e}^{\mathrm{i} s x}\left(s-\gamma_{2}\right)^{n} \mathrm{~d} s, \\
& \chi_{n}^{(3)}=\mathrm{K}_{n}\left(q_{2} r\right) \mathrm{e}^{\mathrm{i} n \theta}+\frac{1}{2 q_{2}^{n}} \int_{-\infty}^{\infty} C^{(3)} \mathrm{e}^{\gamma_{2}(z-h)} \mathrm{e}^{\mathrm{i} s x}\left(s-\gamma_{2}\right)^{n} \frac{\mathrm{d} s}{\gamma_{2}},
\end{aligned}
$$

$$
=\frac{1}{2 q_{2}^{n}} \int_{-\infty}^{\infty}\left[\mathrm{e}^{-\gamma_{2}(z+h)}+C^{(3)} \mathrm{e}^{\gamma_{2}(z-h)}\right] \mathrm{e}^{\mathrm{i} s x}\left(s-\gamma_{2}\right)^{n} \frac{\mathrm{d} s}{\gamma_{2}}, \quad z>-h,
$$

where $\gamma_{i}=\tilde{\gamma}\left(s ; \mathrm{i} q_{i}\right)=\left(s^{2}+q_{i}^{2}\right)^{1 / 2}$.

For the multipole triple $\left\{\phi_{n}^{(1)}, \psi_{n}^{(1)}, \chi_{n}^{(1)}\right\}$, application of the boundary conditions (2.16), (2.15) and (2.17) yields

$$
\begin{aligned}
& A^{(1)}=-\frac{1}{\Delta}\left(\left(2 \hat{s}^{2}-k_{2}^{2}\right)^{2}+4 \hat{s}^{2} \gamma_{1} \gamma_{2}\right)=-1-\frac{8 \hat{s}^{2} \gamma_{1} \gamma_{2}}{\Delta}, \\
& B^{(1)}=\frac{4 s k_{2}^{2}}{q_{2}^{2} \Delta}\left(2 \hat{s}^{2}-k_{2}^{2}\right), \quad C^{(1)}=-\frac{4 k_{2} \gamma_{2} \ell}{q_{2}^{2} \Delta}\left(2 \hat{s}^{2}-k_{2}^{2}\right),
\end{aligned}
$$


where we have written $\hat{s}^{2}=s^{2}+\ell^{2}$ and

$$
\Delta=\left(2 \hat{s}^{2}-k_{2}^{2}\right)^{2}-4 \hat{s}^{2} \gamma_{1} \gamma_{2}=\left(2 s^{2}-\nu^{2}\right)^{2}-4 \hat{s}^{2} \gamma_{1} \gamma_{2} .
$$

Note that $A^{(1)}(s)$ and $C^{(1)}(s)$ are even functions, whereas $B^{(1)}(s)$ is an odd function. Since $\gamma_{i}^{2}=\hat{s}^{2}-k_{i}^{2}, \Delta$ is the secular determinant for Rayleigh waves with $\Delta=0$ implying

$$
\left(2 \hat{s}^{2}-k_{2}^{2}\right)^{4}=16 \hat{s}^{4}\left(\hat{s}^{2}-k_{1}^{2}\right)\left(\hat{s}^{2}-k_{2}^{2}\right),
$$

which simplifies to the cubic

$$
\left(k_{2}^{2} / \hat{s}^{2}\right)^{3}-8\left(k_{2}^{2} / \hat{s}^{2}\right)^{2}+8\left(k_{2}^{2} / \hat{s}^{2}\right)(3-2 \Lambda)-16(1-\Lambda)=0 .
$$

Only the real root (for $k_{2}^{2} / \hat{s}^{2}$ ) lying in $(0,1)$ is actually a solution to $\Delta=0$ (the others, when they exist, correspond to taking different branches in the definition of $\gamma_{i}$ ). This leads to poles in the integrands at $\hat{s}^{2}=k_{\mathrm{R}}^{2}$ or equivalently at $s= \pm \mathrm{i}\left(\ell^{2}-k_{\mathrm{R}}^{2}\right)^{1 / 2}$. Since we have $\ell>k_{\mathrm{R}}$, these poles are not on the real axis.

Similarly for the multipole triple $\left\{\phi_{n}^{(2)}, \psi_{n}^{(2)}, \chi_{n}^{(2)}\right\}$

$$
A^{(2)}=-\frac{4 s}{\Delta}\left(2 \hat{s}^{2}-k_{2}^{2}\right), \quad B^{(2)}=-1+\frac{8 \gamma_{1} \gamma_{2} k_{2}^{2} s^{2}}{q_{2}^{2} \Delta}, \quad C^{(2)}=-\frac{8 \gamma_{1} \gamma_{2} \ell s k_{2}}{q_{2}^{2} \Delta} .
$$

Note that $A^{(2)}(s)$ and $C^{(2)}(s)$ are odd functions, whereas $B^{(2)}(s)$ is an even function. Finally, for the triple $\left\{\phi_{n}^{(3)}, \psi_{n}^{(3)}, \chi_{n}^{(3)}\right\}$ we have

$$
A^{(3)}=\frac{4 \gamma_{2} \ell}{k_{2} \Delta}\left(2 \hat{s}^{2}-k_{2}^{2}\right), \quad B^{(3)}=C^{(2)}, \quad C^{(3)}=1+\frac{8 \gamma_{1} \gamma_{2}^{3} \ell^{2}}{q_{2}^{2} \Delta},
$$

with $A^{(3)}(s)$ and $C^{(3)}(s)$ even and $B^{(3)}(s)$ odd.

A.2. Polar expansions. Polar expansions of the multipoles can be obtained as follows. Let $v_{i}=\sinh ^{-1}\left(s / q_{i}\right)$ so that

$$
\mathrm{e}^{v_{i}}=\left(\gamma_{i}+s\right) / q_{i}, \quad \mathrm{e}^{-v_{i}}=\left(\gamma_{i}-s\right) / q_{i} .
$$

Then,

$$
\mathrm{e}^{\gamma_{i} z} \mathrm{e}^{\mathrm{i} s x}=\mathrm{e}^{-\gamma_{i} h} \sum_{n=-\infty}^{\infty}(-1)^{n} \mathrm{I}_{n}\left(q_{i} r\right) \mathrm{e}^{\mathrm{i} n \theta} \mathrm{e}^{-n v_{i}}
$$

Hence

$$
\begin{gathered}
\phi_{n}^{(1)}=\mathrm{K}_{n}\left(q_{1} r\right) \mathrm{e}^{\mathrm{i} n \theta}+\sum_{m=-\infty}^{\infty} A_{n m}^{(1)} \mathrm{I}_{m}\left(q_{1} r\right) \mathrm{e}^{\mathrm{i} m \theta}, \\
\psi_{n}^{(1)}=\mathrm{i} \sum_{m=-\infty}^{\infty} B_{n m}^{(1)} \mathrm{I}_{m}\left(q_{2} r\right) \mathrm{e}^{\mathrm{i} m \theta}, \quad \chi_{n}^{(1)}=\mathrm{i} \sum_{m=-\infty}^{\infty} C_{n m}^{(1)} \mathrm{I}_{m}\left(q_{2} r\right) \mathrm{e}^{\mathrm{i} m \theta},
\end{gathered}
$$

where

$$
\begin{gathered}
A_{n m}^{(1)}=\frac{(-1)^{n+m}}{2} \int_{-\infty}^{\infty} A^{(1)} \mathrm{e}^{-2 \gamma_{1} h} \mathrm{e}^{-(m+n) v_{1}} \frac{\mathrm{d} s}{\gamma_{1}}, \\
B_{n m}^{(1)}=\frac{(-1)^{n+m}}{2} \int_{-\infty}^{\infty} B^{(1)} \mathrm{e}^{-\left(\gamma_{1}+\gamma_{2}\right) h} \mathrm{e}^{-m v_{2}-n v_{1}} \mathrm{~d} s, \\
C_{n m}^{(1)}=\frac{(-1)^{n+m}}{2} \int_{-\infty}^{\infty} C^{(1)} \mathrm{e}^{-\left(\gamma_{1}+\gamma_{2}\right) h} \mathrm{e}^{-m v_{2}-n v_{1}} \mathrm{~d} s .
\end{gathered}
$$




$$
\begin{gathered}
\phi_{n}^{(2)}=-\mathrm{i} \sum_{m=-\infty}^{\infty} A_{n m}^{(2)} \mathrm{I}_{m}\left(q_{1} r\right) \mathrm{e}^{\mathrm{i} m \theta}, \quad \chi_{n}^{(2)}=\sum_{m=-\infty}^{\infty} C_{n m}^{(2)} \mathrm{I}_{m}\left(q_{2} r\right) \mathrm{e}^{\mathrm{i} m \theta}, \\
\psi_{n}^{(2)}=\mathrm{K}_{n}\left(q_{2} r\right) \mathrm{e}^{\mathrm{i} n \theta}+\sum_{m=-\infty}^{\infty} B_{n m}^{(2)} \mathrm{I}_{m}\left(q_{2} r\right) \mathrm{e}^{\mathrm{i} m \theta},
\end{gathered}
$$

$$
\begin{gathered}
A_{n m}^{(2)}=\frac{(-1)^{n+m}}{2} \int_{-\infty}^{\infty} A^{(2)} \mathrm{e}^{-\left(\gamma_{1}+\gamma_{2}\right) h} \mathrm{e}^{-m v_{1}-n v_{2}} \mathrm{~d} s \\
B_{n m}^{(2)}=\frac{(-1)^{n+m}}{2} \int_{-\infty}^{\infty} B^{(2)} \mathrm{e}^{-2 \gamma_{2} h} \mathrm{e}^{-(m+n) v_{2}} \frac{\mathrm{d} s}{\gamma_{2}} \\
C_{n m}^{(2)}=\frac{(-1)^{n+m}}{2} \int_{-\infty}^{\infty} C^{(2)} \mathrm{e}^{-2 \gamma_{2} h} \mathrm{e}^{-(m+n) v_{2}} \mathrm{~d} s
\end{gathered}
$$

$$
\begin{gathered}
A_{n m}^{(3)}=\frac{(-1)^{n+m}}{2} \int_{-\infty}^{\infty} A^{(3)} \mathrm{e}^{-\left(\gamma_{1}+\gamma_{2}\right) h} \mathrm{e}^{-m v_{1}-n v_{2}} \mathrm{~d} s, \quad B_{n m}^{(3)}=C_{n m}^{(2)}, \\
C_{n m}^{(3)}=\frac{(-1)^{n+m}}{2} \int_{-\infty}^{\infty} C^{(3)} \mathrm{e}^{-2 \gamma_{2} h} \mathrm{e}^{-(m+n) v_{2}} \frac{\mathrm{d} s}{\gamma_{2}} .
\end{gathered}
$$

$$
\begin{gathered}
\phi_{n}^{(3)}=-\mathrm{i} \sum_{m=-\infty}^{\infty} A_{n m}^{(3)} \mathrm{I}_{m}\left(q_{1} r\right) \mathrm{e}^{\mathrm{i} m \theta}, \quad \psi_{n}^{(3)}=\sum_{m=-\infty}^{\infty} B_{n m}^{(3)} \mathrm{I}_{m}\left(q_{2} r\right) \mathrm{e}^{\mathrm{i} m \theta}, \\
\chi_{n}^{(3)}=\mathrm{K}_{n}\left(q_{2} r\right) \mathrm{e}^{\mathrm{i} n \theta}+\sum_{m=-\infty}^{\infty} C_{n m}^{(3)} \mathrm{I}_{m}\left(q_{2} r\right) \mathrm{e}^{\mathrm{i} m \theta},
\end{gathered}
$$

where

Note that changing $s$ to $-s$ shows that

$$
A_{n m}^{(i)}=(-1)^{i+1} A_{-n,-m}^{(i)}, \quad B_{n m}^{(i)}=(-1)^{i} B_{-n,-m}^{(i)}, \quad C_{n m}^{(i)}=(-1)^{i+1} C_{-n,-m}^{(i)} .
$$

A.3. Behaviour as $\ell \rightarrow k_{\mathrm{R}}$. If we set $\ell^{2}=k_{\mathrm{R}}^{2}+\epsilon^{2}, \epsilon>0$, then the integrands above have poles at $s= \pm \mathrm{i} \epsilon$. Thus if we let $\epsilon \rightarrow 0$, the multipoles will have singularities due to these poles 'pinching' the integration contour. In order to determine the behaviour of the multipoles in this limit we lift the contour off the real axis and pick up a contribution from the pole at $s=\mathrm{i} \epsilon$. We will write $\widehat{A}_{n m}^{(i)}, \widehat{B}_{n m}^{(i)}$ and $\widehat{C}_{n m}^{(i)}$ for the expressions in (A.23), (A.25) and (A.27) with this shifted contour.

Some of the multipoles are regular in this limit; as an example consider $B_{n m}^{(1)}$. Since $\gamma_{i}=\beta_{i}$ when $s=\mathrm{i} \epsilon$, we find that

$$
B_{n m}^{(1)}=\widehat{B}_{n m}^{(1)}-\frac{4 \pi \epsilon k_{2}^{2}\left(2 k_{\mathrm{R}}^{2}-k_{2}^{2}\right)}{q_{2}^{2} \Delta^{\prime}(\mathrm{i} \epsilon)}\left(\frac{\mathrm{i} \epsilon-\beta_{2}}{q_{2}}\right)^{m}\left(\frac{\mathrm{i} \epsilon-\beta_{1}}{q_{1}}\right)^{n} \mathrm{e}^{-\left(\beta_{1}+\beta_{2}\right) h},
$$

where, from (A.15),

$$
\Delta^{\prime}(s)=4 s\left[4 s^{2}-2 \nu^{2}-2 \gamma_{1} \gamma_{2}-\left(s^{2}+\ell^{2}\right)\left(\frac{\gamma_{1}}{\gamma_{2}}+\frac{\gamma_{2}}{\gamma_{1}}\right)\right] .
$$


Note that, from (A.16),

$$
2 k_{\mathrm{R}}^{2}-k_{2}^{2}=2 k_{\mathrm{R}} \sqrt{\beta_{1} \beta_{2}} .
$$

The singularity as $\epsilon \rightarrow 0$ is therefore removable and

$$
\left.B_{n m}^{(1)}\right|_{\epsilon=0}=\left.\widehat{B}_{n m}^{(1)}\right|_{\epsilon=0}-\frac{2 \pi \mathrm{i} k_{2}^{2} k_{\mathrm{R}} \sqrt{\beta_{1} \beta_{2}}}{q_{2}^{2} p_{0}}\left(\frac{-\beta_{2}}{q_{2}}\right)^{m}\left(\frac{-\beta_{1}}{q_{1}}\right)^{n} \mathrm{e}^{-\left(\beta_{1}+\beta_{2}\right) h},
$$

where

$$
p_{0}=2 \nu^{2}+2 \beta_{1} \beta_{2}+k_{\mathrm{R}}^{2}\left(\frac{\beta_{1}}{\beta_{2}}+\frac{\beta_{2}}{\beta_{1}}\right) .
$$

Furthermore, $B_{n m}^{(1)}$ is real for all $\epsilon>0$ and hence

$$
\left.B_{n m}^{(1)}\right|_{\epsilon=0}=\left.\operatorname{Re} \widehat{B}_{n m}^{(1)}\right|_{\epsilon=0} .
$$

The terms $A_{n m}^{(2)}, B_{n m}^{(1)}, B_{n m}^{(2)}, B_{n m}^{(3)}$ and $C_{n m}^{(2)}$ are all regular as $\epsilon \rightarrow 0$ and can be treated in a similar way.

Next, consider $A_{n m}^{(1)}$. We have

$$
A_{n m}^{(1)}=\widehat{A}_{n m}^{(1)}-\frac{8 \pi \mathrm{i} \beta_{2} k_{\mathrm{R}}^{2}}{\Delta^{\prime}(\mathrm{i} \epsilon)}\left(\frac{\mathrm{i} \epsilon-\beta_{1}}{q_{1}}\right)^{m+n} \mathrm{e}^{-2 \beta_{1} h}
$$

and hence, since $q_{i}=\beta_{i}+O\left(\epsilon^{2}\right)$,

(A.36) $A_{n m}^{(1)} \sim \widehat{A}_{n m}^{(1)}+(-1)^{m+n} \frac{2 \pi \beta_{2} k_{\mathrm{R}}^{2}}{\epsilon p_{0}}\left(1-\frac{\mathrm{i} \epsilon(m+n)}{\beta_{1}}\right) \mathrm{e}^{-2 \beta_{1} h}+O(\epsilon) \quad$ as $\epsilon \rightarrow 0$.

Since $A_{n m}^{(1)}$ is real for all $\epsilon>0$, we must have

$$
A_{n m}^{(1)} \sim(-1)^{m+n} \frac{a^{(1)}}{\epsilon}+\operatorname{Re} \widehat{A}_{n m}^{(1)}+O(\epsilon) \quad \text { as } \epsilon \rightarrow 0,
$$

where

$$
a^{(1)}=\frac{2 \pi \beta_{2} k_{\mathrm{R}}^{2}}{p_{0}} \mathrm{e}^{-2 \beta_{1} h} .
$$

Similarly

$$
\begin{aligned}
& C_{n m}^{(1)} \sim(-1)^{m+n} \frac{c^{(1)}}{\epsilon}+\operatorname{Re} \widehat{C}_{n m}^{(1)}+O(\epsilon), \\
& A_{n m}^{(3)} \sim(-1)^{m+n} \frac{a^{(3)}}{\epsilon}+\operatorname{Re} \widehat{A}_{n m}^{(3)}+O(\epsilon), \\
& C_{n m}^{(3)} \sim(-1)^{m+n} \frac{c^{(3)}}{\epsilon}+\operatorname{Re} \widehat{C}_{n m}^{(3)}+O(\epsilon),
\end{aligned}
$$

as $\epsilon \rightarrow 0$, where

$$
\begin{aligned}
& c^{(1)}=\frac{2 \pi k_{2} k_{\mathrm{R}}^{2} \sqrt{\beta_{1} \beta_{2}}}{p_{0} \beta_{2}} \mathrm{e}^{-\left(\beta_{1}+\beta_{2}\right) h}, \\
& a^{(3)}=-\frac{2 \pi \beta_{2} k_{\mathrm{R}}^{2} \sqrt{\beta_{1} \beta_{2}}}{p_{0} k_{2}} \mathrm{e}^{-\left(\beta_{1}+\beta_{2}\right) h}, \\
& c^{(3)}=-\frac{2 \pi \beta_{1} k_{\mathrm{R}}^{2}}{p_{0}} \mathrm{e}^{-2 \beta_{2} h} .
\end{aligned}
$$


Note that

$$
\frac{a^{(1)}}{a^{(3)}}=\frac{c^{(1)}}{c^{(3)}}=-\frac{k_{2}}{\sqrt{\beta_{1} \beta_{2}}} \mathrm{e}^{\left(\beta_{2}-\beta_{1}\right) h}
$$

In a similar way we can determine the behaviour of the functions $\phi_{n}^{(i)}, \psi_{n}^{(i)}$ and $\chi_{n}^{(i)}$ in the limit as $\epsilon \rightarrow 0$. We write $\widehat{\phi}_{n}^{(i)}, \widehat{\psi}_{n}^{(i)}$ and $\chi_{n}^{(i)}$ to indicate that the contour of integration is shifted above the pole at $s=\mathrm{i} \epsilon$ and then we find that, for fixed $x>0$ (the case $x<0$ can always be determined through symmetry considerations),

$$
\begin{aligned}
& \phi_{n}^{(1)}=\widehat{\phi}_{n}^{(1)}+(-1)^{n} \frac{2 \pi k_{\mathrm{R}}^{2} \beta_{2}}{\epsilon p_{0}} \mathrm{e}^{\beta_{1}(z-h)}\left(1-x \epsilon-\mathrm{i} n \epsilon / \beta_{1}\right)+O(\epsilon) \\
& \psi_{n}^{(1)}=\widehat{\psi}_{n}^{(1)}+(-1)^{n} \frac{2 \pi}{p_{0}} k_{\mathrm{R}} k_{2}^{2} \beta_{1}^{1 / 2} \beta_{2}^{-3 / 2} \mathrm{e}^{\beta_{2} z} \mathrm{e}^{-\beta_{1} h}+O(\epsilon)
\end{aligned}
$$

and

$$
\chi_{n}^{(1)}=\widehat{\chi}_{n}^{(1)}+(-1)^{n} \frac{2 \pi \mathrm{i}}{\epsilon p_{0}} k_{\mathrm{R}}^{2} k_{2} \beta_{1}^{1 / 2} \beta_{2}^{-1 / 2} \mathrm{e}^{\beta_{2} z} \mathrm{e}^{-\beta_{1} h}\left(1-x \epsilon-\mathrm{i} n \epsilon / \beta_{1}\right)+O(\epsilon),
$$

having used (A.31). For the second set of multipoles, we obtain

$$
\begin{aligned}
& \phi_{n}^{(2)}=\widehat{\phi}_{n}^{(2)}+(-1)^{n} \frac{2 \pi}{p_{0}} k_{\mathrm{R}} \beta_{1}^{1 / 2} \beta_{2}^{1 / 2} \mathrm{e}^{\beta_{1} z} \mathrm{e}^{-\beta_{2} h}+O(\epsilon), \\
& \psi_{n}^{(2)}=\widehat{\psi}_{n}^{(2)}+O(\epsilon)
\end{aligned}
$$

and

$$
\chi_{n}^{(2)}=\widehat{\chi}_{n}^{(2)}+(-1)^{n} \frac{2 \pi \mathrm{i}}{p_{0}} k_{\mathrm{R}} k_{2} \beta_{1} \beta_{2}^{-1} \mathrm{e}^{\beta_{2}(z-h)}+O(\epsilon) .
$$

For the final set, we observe that $\psi_{n}^{(3)}=\chi_{n}^{(2)}$, so we need only deal with $\phi_{n}^{(3)}$ and $\chi_{n}^{(3)}$. We find that

$$
\phi_{n}^{(3)}=\widehat{\phi}_{n}^{(3)}+(-1)^{n} \frac{2 \pi \mathrm{i}}{\epsilon p_{0}} k_{\mathrm{R}}^{2} k_{2}^{-1} \beta_{1}^{1 / 2} \beta_{2}^{3 / 2} \mathrm{e}^{\beta_{1} z} \mathrm{e}^{-\beta_{2} h}\left(1-x \epsilon-\mathrm{i} n \epsilon / \beta_{2}\right)+O(\epsilon)
$$

and

$$
\chi_{n}^{(3)}=\widehat{\chi}_{n}^{(3)}-(-1)^{n} \frac{2 \pi}{\epsilon p_{0}} k_{\mathrm{R}}^{2} \beta_{1} \mathrm{e}^{\beta_{2}(z-h)}\left(1-x \epsilon-\mathrm{i} n \epsilon / \beta_{2}\right)+O(\epsilon) .
$$

\section{REFERENCES}

[1] J. A. P. Aranha, Existence and some properties of waves trapped by a submerged cylinder, J. Fluid Mech., 192 (1988), pp. 421-433, https://doi.org/10.1017/S0022112088001922.

[2] A. Bamberger, Y. Dermenjian, and P. Joly, Mathematical analysis of the propagation of elastic guided waves in heterogeneous media, J. Differential Equations, 88 (1990), pp. 113154, https://doi.org/10.1016/0022-0396(90)90112-3.

[3] A. Bamberger, P. Joly, And M. Kern, Propagation of elastic surface waves along a cylindrical cavity of arbitrary cross section, RAIRO - Modélisation mathématique et analyse numérique, 25 (1991), pp. 1-30.

[4] M. A. Biot, Propagation of elastic waves in a cylindrical bore containing a fluid, J. Appl. Phys., 23 (1952), pp. 997-1005, https://doi.org/10.1063/1.1702365.

[5] A. Boström And A. Burden, Propagation of elastic surface waves along a cylindrical cavity and their excitation by a point force, J. Acoust. Soc. Am., 72 (1982), pp. 998-1004, https:// doi.org/10.1121/1.388182.

[6] A. D. BURDEN, The propagation of elastic surface waves along cylindrical cavities of general cross section, Wave Motion, 7 (1985), pp. 153-168, https://doi.org/10.1016/ 0165-2125(85)90043-5. 
[7] K. D. Cherednichenko And F. J. Sabina, On the existence of waves guided by a cavity in an elastic film, Q. J. Mech. Appl. Math., 62 (2009), pp. 221-233, https://doi.org/10.1093/ qjmam/hbp007.

[8] H. Fossen, Structural Geology, Cambridge University Press, 2010.

[9] H. Gercek, Poisson's ratio values for rocks, International Journal of Rock Mechanics and Mining Sciences, 44 (2007), pp. 1-13, https://doi.org/10.1016/j.ijrmms.2006.04.011.

[10] K. F. Graff, Wave Motion in Elastic Solids, Oxford University Press, 1975.

[11] D. S. Jones, The eigenvalues of $\nabla^{2} u+\lambda u=0$ when the boundary conditions are given on semi-infinite domains, Proc. Camb. Phil. Soc., 49 (1953), pp. 668-684, https://doi.org/10. 1017/S0305004100028875.

[12] W. I. LiaO, C. S. Yeh, And T. J. Teng, Scattering of elastic waves by a buried tunnel under obliquely incident waves using T matrix, Chinese Journal of Mechanics, A, 24 (2008), pp. 405-418, https://doi.org/10.1017/S1727719100002525.

[13] C. M. Linton And I. Thompson, Oblique Rayleigh wave scattering by a cylindrical cavity, Q. J. Mech. Appl. Math., 68 (2015), pp. 235-261, https://doi.org/10.1093/qjmam/hbv006.

[14] P. MCIVER AND D. V. Evans, The trapping of surface waves above a submerged horizontal cylinder, J. Fluid Mech., 151 (1985), pp. 243-255, https://doi.org/10.1017/ S0022112085000945.

[15] I. A. Minduin, Free elastic waves on the surface of a tube of infinite thickness, J. Appl. Maths Mechs, 27 (1963), pp. 823-828, https://doi.org/10.1016/0021-8928(63)90165-5.

[16] NIST Handbook of Mathematical Functions, NIST and Cambridge University Press, 2010.

[17] A. M. Turing, A method for the calculation of the zeta-function, Proceedings of the London Mathematical Society, 48 (1945), pp. 180-197, https://doi.org/10.1112/plms/s2-48.1.180.

[18] F. Unsell, Trapping modes in the theory of surface waves, Proc. Camb. Phil. Soc., 47 (1951), pp. 347-358, https://doi.org/https://doi.org/10.1017/S0305004100026700.

[19] F. URsell, Mathematical aspects of trapping modes in the theory of surface waves, J. Fluid Mech., 183 (1987), pp. 421-437, https://doi.org/https://doi.org/10.1017/ S0022112087002702.

[20] I. A. Viktonov, Rayleigh and Lamb waves. Physical Theory and Applications, Plenum Press, New York, 1967.

[21] Z. Yuan, A. Boström, AND Y. CAI, Benchmark solution for vibrations from a moving point source in a tunnel embedded in a half-space, J. Sound Vib., 387 (2017), pp. 177-193, https://doi.org/10.1016/j.jsv.2016.10.016. 